\title{
Ab Initio Magneto-Optical Spectrum of Group-IV Vacancy Color Centers in Diamond
}

\author{
Gergő Thiering and Adam Gali* \\ Wigner Research Centrefor Physics, Hungarian Academy of Sciences, PO Box 49, H-1525 Budapest, Hungary \\ and Department of Atomic Physics, Budapest University of Technology and Economics, \\ Budafoki út 8, H-1111 Budapest, Hungary
}

(Received 6 November 2017; revised manuscript received 18 April 2018; published 11 June 2018)

\begin{abstract}
Group-IV vacancy color centers in diamond are fast emerging qubits that can be harnessed in quantum communication and sensor applications. There is an immediate quest for understanding their magnetooptical properties, in order to select the appropriate qubits for varying needs of particular quantum applications. Here we present results from cutting-edge $a b$ initio calculations about the charge state stability, zero-phonon-line energies, and spin-orbit and electron-phonon couplings for group-IV vacancy color centers. Based on the analysis of our results, we develop a novel spin Hamiltonian for these qubits which incorporates the interaction of the electron spin and orbit coupled with phonons beyond perturbation theory. Our results are in good agreement with previous data and predict a new defect for qubit applications with thermally initialized ground state spin and long spin coherence time.

DOI: 10.1103/PhysRevX.8.021063

Subject Areas: Condensed Matter Physics,

Quantum Physics
\end{abstract}

\section{INTRODUCTION}

In recent years, vacancy-impurity defects in diamond have become of high interest and are important because they show great potential in various quantum technology applications. In particular, the spin properties of the negatively charged silicon-vacancy $[\mathrm{SiV}(-)]$ color center [1-4] with a zero-phonon-line (ZPL) energy at $1.682 \mathrm{eV}$ and $S=1 / 2$ spin has recently been studied for qubit applications [5-10]. As this defect has inversion symmetry ( $D_{3 d}$ point group), it does not directly couple to an external electric field, and as a consequence, $\operatorname{SiV}(-)$ possesses narrow [11] inhomogeneous linewidth and negligible spectral diffusion [7,8]. In addition, $\sim 70 \%$ of the total emission occurs in ZPL emission [5], with a corresponding Huang-Rhys (HR) factor of 0.3. These properties are promising for realizing solid-state sources of indistinguishable single photons for quantum communication applications [8]. The fine splitting in the ground and excited levels caused by spin-orbit coupling (SOC) is harnessed to realize $\Lambda$ scheme qubit operation [12]; however, the spin coherence time of $35 \mathrm{~ns}$ (see Refs. [7,13]) is short because of the fast scattering of the electrons between the sublevels in the ground state mediated by the dynamic Jahn-Teller (DJT)

\footnotetext{
*gali.adam@wigner.mta.hu
}

Published by the American Physical Society under the terms of the Creative Commons Attribution 4.0 International license. Further distribution of this work must maintain attribution to the author(s) and the published article's title, journal citation, and DOI. effect even at $T=4.5 \mathrm{~K}$. Improvement on the spin coherence time can be achieved by cooling down the system below $T=0.5 \mathrm{~K}[14,15]$, which suppresses the density states of the phonons that can mediate this process. Let us mention here that $\mathrm{SiV}(-)$ has also been proposed for optical thermometry at the nanoscale using the temperature-dependent shift of the ZPL [16]. We further note that the neutral $\mathrm{SiV}, \mathrm{SiV}(0)$, with a ZPL at $1.31 \mathrm{eV}$ and $S=1$ spin [17], exhibits spin coherence time almost up to 1 second [18] and relaxation time nearly 1 minute [18]. $\mathrm{SiV}(0)$ is associated with the KUL1 electron paramagnetic resonance (EPR) center $[4,17,19,20]$. We note that $\mathrm{SiV}(0)$ can be found in special diamond samples where boron and silicon doping should be simultaneously realized during the diamond chemical vapor deposition (CVD) growth [18].

Inspired by the success of the $\mathrm{SiV}(-)$ color center, germanium, another group-IV element in the periodic table, was introduced into the diamond lattice, either by CVD growth [21-23] or high-pressure high-temperature synthesis [24-27]. In all samples, a new ZPL line at $602 \mathrm{~nm}$ $(2.06 \mathrm{eV})$ was observed in the PL spectrum, and unambiguously identified as a Ge-related center because of the isotopic shift in the ZPL line [24], its first vibronic peak [25], and the anharmonicity of this peak [28] in the PL spectrum. Theory predicted $[21,29]$ that this defect possesses the same $D_{3 d}$ symmetry as $\mathrm{SiV}$ does. The observed optical transitions support this conclusion [21,24]; thus, the defect is indeed $\mathrm{GeV}(-)$. The 2.06-eV line has narrow $(5 \mathrm{~nm})$ ZPL linewidth even at room temperature, and a short excited state lifetime [30] (6-7 ns). The majority of the emission is concentrated in the ZPL emission [24], with a 
HR factor of 0.5 . Very recently, the spin relaxation and coherence times have been observed in Ge-doped diamond samples at $T_{1} \sim 0.34-25 \mu \mathrm{s}$ and $T_{2} \sim 19$ ns (see Ref. [27]), respectively, at $T=2 \mathrm{~K}$. We note that the signatures of $\mathrm{GeV}(0)$ with $S=1$ spin have been observed in EPR spectrum in high-pressure high-temperature diamond [31,32]. This defect might have improved spin properties similar to those of $\mathrm{SiV}(0)$. The optical signature of $\mathrm{GeV}(0)$ has not yet been identified.

Recently, Sn-related PL centers have been reported in Sn implanted diamond [33,34], where $\mathrm{Sn}$ is another group-IV element next to germanium in the periodic table. In particular, the 620.3-nm PL signal showed the same fine level structure in PL like that of $\operatorname{SiV}(-)$ and $\mathrm{GeV}(-)$ [34]. By the use of this analog, they concluded that the 620.3-nm center is associated with $\mathrm{SnV}(-)$. The spin properties and other charge states of this defect have not been reported. Next to tin, lead is the next group-IV element in the periodic table. To the best of our knowledge, no $\mathrm{Pb}$-related color centers have been reported in diamond so far.

Understanding the magneto-optical properties and spin coherence time of group-IV vacancy color centers is of immediate interest and high importance in the fast emerging field of solid-state qubits. Here we present a systematic study on the magneto-optical properties of the group-IV vacancy defects, including $\mathrm{Si}, \mathrm{Ge}, \mathrm{Sn}$, and $\mathrm{Pb}$ impurities, by means of cutting-edge first-principles methods. In Sec. II, we describe the first-principles methodology for calculating the electronic structure, spin-orbit interaction, and electronphonon coupling of the systems. We then continue with a detailed description of the results in Sec. III, where we discuss the photostability and spin Hamiltonian of the qubits. We find that the $\mathrm{PbV}$ color center exhibits superior spin properties over the other group-IV vacancy color centers. Finally, we conclude the results in Sec. IV. We give additional data and derivation on the developed spin Hamiltonian in the Appendixes.

\section{METHODS}

We characterize point defects embedded in diamond within spin-polarized density functional theory (DFT) as implemented in the VASP.5.4.1 code [35]. Our DFT method is within the Born-Oppenheimer approximation, as ions are treated as classical particles. By varying the positions of ions one can achieve an adiabatic potential energy surface (APES) map of the system. The global minimum of APES defines the optimized geometry of the system. We reach this minimum upon relaxing the atomic positions until the force acting on every ion falls below $10^{-2} \mathrm{eV} / \AA$. We embed the point defects into a 512-atom diamond supercell, which suffices to sample the Brillouin zone only at the $\Gamma$ point for converged charged density. A relatively low-energy cutoff $(370 \mathrm{eV})$ for the expansion of the plane waves within the applied projector-augmentation-wave method (PAW) [36,37] yields converged results. We calculated the excited states with the constrained-occupation DFT method (CDFT) [38]. We relaxed the atomic positions by minimizing the forces acting on them in the excited electronic state within the CDFT method. The electronic structure is calculated using the HSE06 hybrid functional $[39,40]$ within DFT. This technique reproduces the experimental band gap and the charge transition levels in group-IV semiconductors within $0.1 \mathrm{eV}$ accuracy [41]. This procedure also yields excellent results for the zero-phonon-line energy of $\mathrm{SiV}(-)$ center in diamond [4]. We determine the adiabatic charge transition levels or photoionization energy thresholds as

$$
E(q \mid q+1)=E_{\mathrm{tot}}^{q}-E_{\mathrm{tot}}^{q+1}+\Delta E_{\mathrm{corr}}^{q}-\Delta E_{\mathrm{corr}}^{q+1},
$$

where $\Delta E^{q}$ is the total energy correction for the point defect with $q$ charge by following the procedure of Lany and Zunger [42] based on the work of Makov and Payne [43], while $E_{\mathrm{tot}}^{q}$ is the total energy of the system including the ions and electrons. We provide these charge transition levels graphically with respect to the valence band (VB) maximum in Fig. 1.
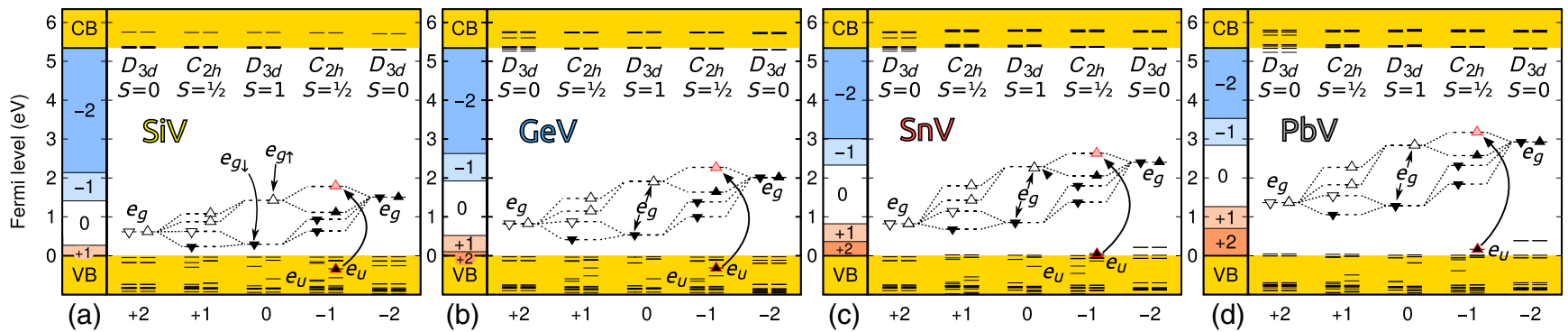

FIG. 1. Kohn-Sham single particle levels and charge transition levels of $X \mathrm{~V}$ defects for $X=\mathrm{Si}, \mathrm{Ge}, \mathrm{Sn}$, and $\mathrm{Pb}$ in (a)-(d), respectively. We note that the effect of spin-orbit interaction is not included here. We represent the $\uparrow(\downarrow)$ spin channel with triangles pointing upwards (downwards). The filled (empty) triangles depict occupied one electron (hole) orbitals. In the (+) and (-) charge states, the symmetry is lowered from $D_{3 d}$ to $C_{2 h}$ within adiabatic potential energy surface in the DFT calculations. We show the charge stability window for the given charge state referenced to the valence band maximum of diamond at the left side in each figure, where the conduction band (CB) is also depicted. The excitation processes are also shown for $X \mathrm{~V}(-)$ color centers. The $e_{u}$ orbital is resonant with the valence band that pops up in the excited electron configuration. 
For the calculation of the phonon sideband of the PL spectrum, we calculated the vibration modes of the defects in a quasiharmonic approximation in the ground state at high symmetry, with equal occupation of the degenerate orbitals in the band gap. We used the numerical derivatives of the forces to generate the Hessian matrix that we diagonalized to obtain the phonon frequencies and normal modes. The geometry is preoptimized with the very strict force criterion of $10^{-4} \mathrm{eV} / \AA$ for the vibration calculations. We consistently applied the computationally powerful Perdew-Burke-Ernzerhof (PBE) functional [44] in this procedure that reproduces the calculated HSE06 phonon spectrum of the perfect diamond within $5 \mathrm{~cm}^{-1}$ and the quasilocal modes of $\mathrm{SiV}(-)$ within $2 \mathrm{~cm}^{-1}$.

We determined the SOC in the noncollinear approach as implemented in VASP.5.4.1 for the negatively charged groupIV vacancy centers, or briefly, $X \mathrm{~V}$ centers. We set the quantization axis of the spin along the $\langle 111\rangle$ direction, the $C_{3}$ rotation axis of the $X \mathrm{~V}$ point defects. We determined the SOC parameters by the HSE06 DFT functional, which has generally provided accurate results for the spin-orbit splitting of the nitrogen-vacancy center in diamond [45]. SOC is a small perturbation to the electronic structure of the system; thus, we fixed the atomic positions in the highsymmetry $D_{3 d}$ configuration as obtained from the previous spin-polarized DFT geometry relaxations. As was reported in our previous study [4] for the $\mathrm{SiV}$ center and is also shown in Sec. II for other $X \mathrm{~V}$ centers, a double degenerate $e_{g\{x, y\}}$ level appears in the gap, which will be occupied by three electrons in the negatively charged state, which can be treated as a single hole on this state. After applying the SOC on the system, $e_{g \pm}=(1 / \sqrt{2})\left(e_{g x} \pm i e_{g y}\right)$ states split by $\lambda_{0}$ (see Fig. 1 for the electronic structure) which comes from the $z$ component of SOC. By using the CDFT procedure it is feasible to introduce the hole on either the $e_{g+}$ or $e_{g-}$ state, and the calculated total energy difference is the strength of spin-orbit coupling. The Hamiltonian of SOC coupling is the following:

$$
\hat{H}_{\mathrm{SOC}}=-\lambda_{0} \hat{L}_{z} \hat{S}_{z}=-\frac{\lambda_{0}}{2}\left[\left|e_{g+}^{\uparrow}\right\rangle\left\langle e_{g+}^{\uparrow}|+| e_{g-}^{\downarrow}\right\rangle\left\langle e_{g-}^{\downarrow}|-| e_{g-}^{\uparrow}\right\rangle\left\langle e_{g-}^{\uparrow}|-| e_{g+}^{\downarrow}\right\rangle\left\langle e_{g+}^{\downarrow}\right|\right],
$$

where $\hat{L}_{z}$ is the effective orbital moment operator $(L=1)$ of the electron, while $\hat{S}_{z}$ is the electronic spin. We find that half of this total energy difference is equal within $10^{-7} \mathrm{eV}$ to the split of $e_{g+}$ and $e_{g-}$ Kohn-Sham levels when these two states are occupied by half-half electrons. Thus, the strength of SOC can be calculated by the half-half occupation of the $e$ states with following the SOC splitting of these $e_{g}$ states. The negative sign of $\lambda_{0}$ accounts for the fact that the $e_{g}$ particle is a hole and not an electron. The SOC Hamiltonian Eq. (2) can be expressed with the Pauli matrices $\hat{H}_{\mathrm{SOC}}=\left(\lambda_{0} / 2\right) \sigma_{y}$, where

$$
\sigma_{y}=\left(\begin{array}{ll} 
& -i \\
i &
\end{array}\right),
$$

that represent the $e_{g \pm}$ electron states.

We note that the dynamic Jahn-Teller effect quenches the orbital moment, at least partially, known as the Ham effect [46-48]. The intrinsic $\lambda_{0}$ that we calculate directly from Kohn-Sham orbitals is severely reduced by the Ham reduction factor $p$; thus, the $\lambda_{\text {Ham }}=p \lambda_{0}$ reduced value is observed in the experiments.

We now discuss two cases. (i) The electron-phonon coupling manifested as the DJT effect is significantly larger than spin-orbit coupling, so one can solve first the electronphonon system and then calculate the spin-orbit energies as a first perturbation acting on the resultant vibronic wavefunctions. (ii) The electron-phonon coupling and spin-orbit coupling are in the same order of magnitude, so the orbital, spin, and phonon degrees of freedom of the wave function are strongly coupled, which requires the exact diagonalization of the sum of spin-orbit and electronphonon Hamiltonians, respectively. We call the first case the Ham reduction factor solution, whereas the second case we call the exact diagonalization procedure.

We first describe case (i) where we define the electronphonon Hamiltonian caused by the DJT effect. The DJT effect is the interaction of an $E_{g}$-type quasilocalized vibration mode with the $e_{g}$ electron orbital, known as the $E \times e$ DJT system. The Hamiltonian of such a system is the following:

$$
\begin{aligned}
\hat{H}_{\mathrm{DJT}}= & \hbar \omega_{e}\left(a_{x}^{\dagger} a_{x}+a_{y}^{\dagger} a_{y}+1\right)+F\left(\hat{x} \sigma_{z}-\hat{y} \sigma_{x}\right) \\
& +G\left[\left(\hat{x}^{2}-\hat{y}^{2}\right) \sigma_{z}+2 \hat{x} \hat{y} \sigma_{x}\right],
\end{aligned}
$$

where the $a_{x, y}^{\dagger}, a_{x, y}$ are the creation and annihilation operators of the $E_{g}$ vibration mode, respectively. The system is a two-dimensional harmonic oscillator with frequency $\omega_{e}$, where the terms labeled by $F$ and $G$ parameters are the corresponding linear and quadratic part of DJT. The $\sigma_{i}$ operators are two-dimensional Pauli matrices,

$$
\sigma_{z}=\left(\begin{array}{cc}
1 & \\
& -1
\end{array}\right), \quad \sigma_{x}=\left(\begin{array}{ll} 
& 1 \\
1 &
\end{array}\right),
$$


representing the $e_{g x}$ and $e_{g y}$ electrons, respectively. $F$ and $G$ parameters are the values describing the Jahn-Teller distortion through $(\hat{x}, \hat{y})=(1 / \sqrt{2})\left(a_{(x, y)}^{\dagger}+a_{(x, y)}\right)$ operators. $F$ and $G$ parameters can be easily derived after the APES of the DJT system is determined. The energy gain from the symmetry distortion (see Chap. 3.2. in Ref. [47]) to one of the three global minima is $E_{\mathrm{JT}}=\left\{F^{2} /\left[2\left(\hbar \omega_{e}-2 G\right)\right]\right\}$, and the barrier energy separating the these three minima is $\delta_{\mathrm{JT}}=\left[\left(4 E_{\mathrm{JT}} G\right) /\left(\hbar \omega_{e}+2 G\right)\right]$. For $\operatorname{SiV}(-), \mathrm{GeV}(-)$, and to a less extent, for $\mathrm{SnV}(-)$ in the ground state, the energy gain from DJT, i.e., $E_{\mathrm{JT}}$, is orders of magnitude larger than the energy of SOC coupling. Thus, SOC can be evaluated as a perturbation on the DJT ground state wave function, where the electrons and vibrations are entangled. We determine the eigenvalues of the Hamiltonian in Eq. (4) numerically with the following series of expansion:

$$
\left|\tilde{\Psi}_{ \pm}\right\rangle=\sum_{n, m}\left[c_{n m}\left|e_{g \pm}\right\rangle|n, m\rangle+d_{n m}\left|e_{g \mp}\right\rangle|n, m\rangle\right] .
$$

We then express the SOC splitting within perturbation theory as $\lambda_{\text {Ham }}=2\left|\left\langle\tilde{\Psi}_{ \pm}\left|\hat{H}_{\text {SOC }}\right| \tilde{\Psi}_{ \pm}\right\rangle\right|=p \lambda_{0}$. We limit the series expansion of the two-dimensional harmonic oscillator $|n, m\rangle$ up to ten quanta; thus, $n+m \leq 10$. From this expansion, the $p$ reduction factor can be expressed as $p=$ $\sum_{n, m}\left(c_{n m}^{2}-d_{n m}^{2}\right)$ with $c_{n m}$ and $d_{n m}$ expansion coefficients (see Appendix B for derivation).

We next describe case (ii), where DJT and SOC energies are comparable, so the corresponding Hamiltonians in Eqs. (4) and (2) should be added and solved simultaneously with coupled orbital, spin, and phonon wave functions. Here, we expanded the polaronic wave function $\tilde{\Psi}_{\Gamma}$ with the spin degrees of freedom as

$$
\left|\tilde{\Psi}_{\Gamma}\right\rangle=\sum_{n, m}\left[c_{n m}^{\chi}\left|e_{g \pm}\right\rangle|n, m\rangle|\chi\rangle+d_{n m}^{\chi}\left|e_{g \mp}\right\rangle|n, m\rangle|\chi\rangle\right]
$$

and then directly diagonalize the Hamiltonian including the SOC and DJT effects simultaneously, where $\chi$ can be either an $\uparrow$ or $\downarrow$ spin state. This solution represents a coupling between spins and phonons and goes beyond the perturbation theory of SOC acting on the polaronic wave functions. Here, the subindex $\Gamma$ refers to the total angular momentum of the wave function, which is either $\frac{3}{2}$ or $\frac{1}{2}$ (see also Appendix C). We note that the simultaneous treatment of SOC and DJT in $E \times e$ JT systems has been considered only for very small molecules at $a b$ initio level in the literature thus far [49,50]. However, extensive $a b$ initio study on point defects in solids, with a model consisting of hundreds of atoms, has not yet been performed, to the best of our knowledge. As we show below, our methodology is able to reproduce previous experimental data.

\section{RESULTS}

All the group-IV impurities reside in the symmetric splitvacancy configuration in diamond according to our calculations that results in a $D_{3 d}$ symmetry. The split-vacancy configuration may be labeled as $\mathrm{V}-\mathrm{X}-\mathrm{V}$, which implies that the $X$ impurity atom lies at an interstitial position, more precisely, at the inversion point of the diamond lattice halfway between two adjacent vacancies, or briefly, in divacancy. Nevertheless, the quantum optics groups labeled these defects by $X \mathrm{~V}$ in the literature; thus, we consequently used this notation in the Introduction and the rest of the paper. According to this description, this type of defect exhibits an inversion symmetry which adds a parity to the wave functions, odd (ungerade) and even (gerade), labeled by $u$ and $g$, respectively. One can construct the possible single electron orbitals from the defect-molecule model. There are 6 carbon dangling bonds pointing towards the impurity atom, from which 6 orbitals emerge in $D_{3 d}$ symmetry: $a_{1 g}+a_{2 u}+e_{u}+e_{g}$. The corresponding orbitals are filled with 10 valence electrons where 6 electrons come from the dangling bonds and 4 electrons from the impurity atom. As discussed previously for the $\mathrm{SiV}$ defect [4], only the $e_{g}$ orbital appears in the gap which is filled by two electrons with parallel spins in the neutral charge state (see also Fig. 1) whereas the $a_{1 g}$ and $a_{2 u}$ levels fall inside the valence band and the $e_{u}$ level is resonant with it. The same $e_{g}$ orbital occurs in the gap for the other $X \mathrm{~V}$ defects. The in-gap optical excitation involves an $e_{u}$ orbital resonant with the valence band that pops up in the gap in the excited state electronic configuration. Special bound exciton states might occur between the in-gap $\leftrightarrow$ band edge optical excitation that might also lead to photoionization. The electronic structure of the neutral $X \mathrm{~V}$ complexes implies that charge states from $(2+)$ to $(2-)$ can exist depending on the position of the (quasi-)Fermi-level in diamond. Here, we focus our study on the (-) charge state (coherent dark states in the prototype $\mathrm{SiV}(-)$ qubit [10]) and the neutral charge state (optical spin polarization with long spin coherence time of prototype $\operatorname{SiV}(0)$ [18]), i.e., their charge state stability. We provide trends for the magneto-optical properties of the negatively charged $X \mathrm{~V}$ complexes.

\section{A. Charge state stability of the $X V$ defects}

A general trend in the electronic structure of $X \mathrm{~V}$ defects is that the $e_{g}$ level shifts up with increasing atomic number of the group-IV impurity atom (see Fig. 1), which has a consequence on the formation energies and the corresponding adiabatic charge transition levels, too. In order to readily see the trends, the calculated $(-) \rightarrow(0)$ and $(0) \rightarrow(-)$ transition energies are also plotted in Fig. 2, where the first and second transition are associated with promoting an electron from the defect level to the conduction band and from the valence band to the defect level, respectively, but the plot depicts the charge transition levels 


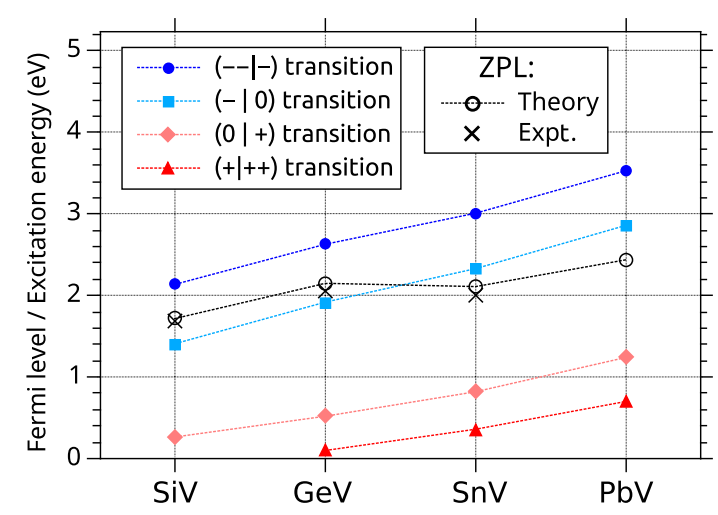

FIG. 2. Adiabatic photoionization of $X \mathrm{~V}$ defects [see Eq. (1)] and excitation energies for $X \mathrm{~V}(-)$ centers (see Sec. III B). All the charge transition levels are referenced to the valence band maximum aligned to zero. The conduction band minimum is at $5.4 \mathrm{eV}$. We note that these energies do not contain spin-orbit energy corrections. See text for explanation.

with respect to the valence band maximum. The stability window of the (-) state shifts up in the gap with increasing atomic number of the group-IV impurity atom. $\mathrm{SnV}(-)$ and $\mathrm{PbV}(-)$ can only be stable by providing substitutional nitrogen donors in the diamond sample. The photostability of $\mathrm{PbV}(-)$ requires special attention as the $(-)$ state can be converted to (0) by illuminating the sample in the visible region, by $\sim 2.6 \mathrm{eV}$, that it is close to its ZPL energy of about $2.4 \mathrm{eV}$ (see Table I). On the other hand, photoexcitation in the visible region may convert the (-) state to the (2-) charge state for $\mathrm{SiV}, \mathrm{GeV}$, and $\mathrm{SnV}$ defects. For isolated $\mathrm{SiV}(-)$, ultraviolet (UV) light would be needed to reionize $(2-)$ to $(-)$ by single photon absorption, which is difficult to realize in experiments. On the other hand, violet and blue illumination can reionize $\mathrm{GeV}(2-)$ and $\mathrm{SnV}(2-)$

TABLE I. The calculated zero-phonon-line (ZPL) energies and HR factors $(S)$ for $X \mathrm{~V}(-)$ defects are given and compared to the experimental data $\left(\mathrm{ZPL}_{\text {expt }}\right.$ and $\left.S_{\text {expt }}\right)$. Here, we provide the average ZPL values in $\mathrm{C}_{2 h}$ symmetry $\left[\mathrm{ZPL}\left(C_{2 h}\right)\right]$ and within exact calculation of dynamic Jahn-Teller (DJT) effect together with spin-orbit coupling [ZPL(SOC)]. The latter more accurate method brings our results closer to the experimental values. See text for explanation.

\begin{tabular}{lcccc}
\hline \hline & $\mathrm{SiV}$ & $\mathrm{GeV}$ & $\mathrm{SnV}$ & $\mathrm{PbV}$ \\
\hline $\mathrm{ZPL}\left(C_{2 h}\right)(\mathrm{eV})$ & 1.72 & 2.15 & 2.11 & 2.45 \\
ZPL(SOC) $(\mathrm{eV})$ & 1.70 & 2.12 & 2.09 & 2.40 \\
$S$ & 0.27 & 0.50 & 0.89 & 1.60 \\
ZPL $_{\text {expt }}(\mathrm{eV})$ & $1.68^{\mathrm{a}}$ & $2.06^{\mathrm{b}}$ & $2.00^{\mathrm{c}}$ & \\
$S_{\text {expt }}$ & $0.24^{\mathrm{d}}$ & $0.50^{\mathrm{e}}$ & $0.89^{\mathrm{c}}$ & \\
\hline \hline
\end{tabular}

${ }^{\mathrm{a}}$ Reference [1].

${ }^{\mathrm{b}}$ References [24,25,51].

${ }^{\mathrm{c}}$ Reference [34].

${ }^{\mathrm{d}}$ References $[52,53]$.

${ }^{\mathrm{e}}$ Reference [24]. to $\mathrm{GeV}(-)$ and $\mathrm{SnV}(-)$, respectively. The (2-) charge state is a spin singlet; nevertheless, a shelving triplet bound exciton state might exist that can be accessed by optical pumping at $\sim 2.4$ and $\sim 1.8 \mathrm{eV}$ for $\mathrm{SnV}(2-)$ and $\mathrm{PbV}(2-)$, respectively. The stability window of the $S=1$ (0) state of $\mathrm{GeV}, \mathrm{SnV}$, and $\mathrm{PbV}$ also shifts up in the gap with increasing the atomic number of the group-IV impurity atom.

One can conclude that the $\mathrm{SnV}(-)$ defect should be very photostable whereas isolated $\mathrm{SiV}$ is trapped in the (2-) charge state once $\mathrm{SiV}(-)$ has been photoionized into that state. On the other hand, there is a small energy margin between the calculated neutral excitation energy $(\sim 2.43 \mathrm{eV})$ and the $(-) \rightarrow(0)$ transition energy $(\sim 2.6 \mathrm{eV})$ for $\mathrm{PbV}(-)$; thus, this defect can be photoionized into the $(0)$ charge state by blue illumination into the phonon sideband.

We note that the neutral $X \mathrm{~V}(0)$ with $S=1$ ground state may also act like a qubit, with presumably long electron spin coherence time in good quality diamond. Our calculations show that $\mathrm{SnV}(0)$ and $\mathrm{PbV}(0)$ defects can be engineered into typical diamond samples where nitrogen contamination occurs, in contrast to the case of $\operatorname{SiV}(0)$ defect, which requires boron doping of diamond. Formation of $\mathrm{GeV}(0)$ requires very low nitrogen concentration or compensation of nitrogen donors by acceptors.

\section{B. Photoluminescence of $X \mathbf{V}(-)$ defects}

The photoluminescence can be described by spontaneous emission from the optically lowest-energy excited state to the ground state. The lowest-energy excited state can be understood as promoting an electron from the $e_{u}$ orbital to the $e_{g}$ orbital in $\mathrm{XV}(-)$ defects. As a consequence, the excited state is ${ }^{2} E_{u}$ and the ground state is ${ }^{2} E_{g}$ in the negatively charged state (see Ref. [4] and Fig. 1). Both ${ }^{2} E_{u}$ and ${ }^{2} E_{g}$ states are dynamic Jahn-Teller systems [4,6,54]. We find a general trend in the calculated Jahn-Teller energy, $E_{\mathrm{JT}}$, as a function of the atomic number of the group-IV impurity atom, where $E_{\mathrm{JT}}$ is defined as the total energy difference in the high-symmetry $D_{3 d}$ geometry and the lowest-energy $C_{2 h}$ geometry in the adiabatic potential energy surface (see Fig. 3). The ZPL energies are calculated by taking the lowest APES energy in $C_{2 h}$ symmetry both in the ground and excited state (see Table I), which we call here an "average" method. Interestingly, the calculations do not predict a linearly increasing trend in the ZPL energies by increasing the atomic number of the group-IV impurity, but the ZPL of $\mathrm{SnV}(-)$ should be smaller than that of $\mathrm{GeV}(-)$. This agrees well with the very recent experimental data [34].

Regarding $X \mathrm{~V}(-)$ qubits or qubit candidates, we show the calculated average ZPL energies compared to the experimental ones when available (see Table I). The calculated average ZPL energies, that do not contain the zero-point energies and spin-orbit couplings in the ground and excited state, somewhat overestimate the experimental 

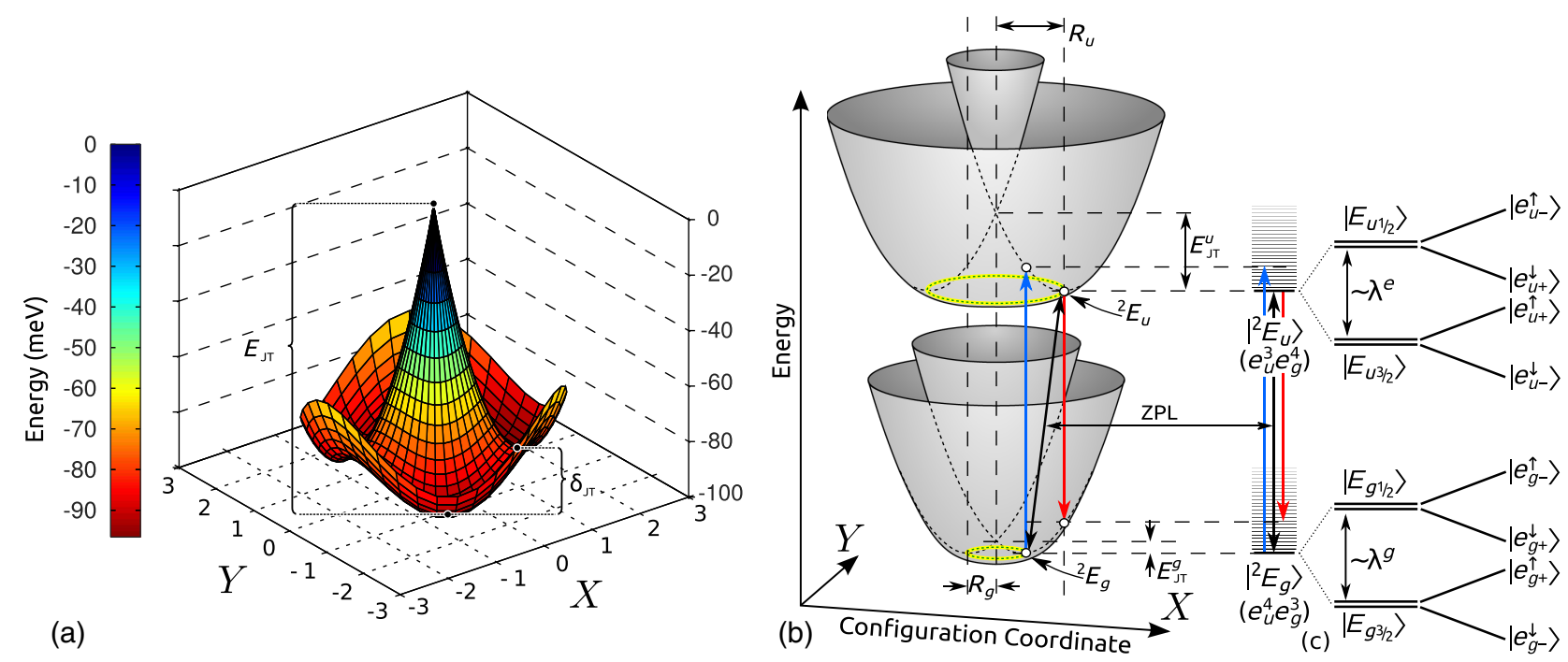

FIG. 3. (a) Jahn-Teller adiabatic potential energy surface (APES) of the optically excited ${ }^{2} E_{u}$ state in $\mathrm{PbV}(-)$. (b) Jahn-Teller APES of $X \mathrm{~V}(-)$ defects as a function of $e_{x}$ and $e_{y}$ phonon distortions, with quadratic JT effects neglected, which results in an APES with axial symmetry. In the ground state $(g)$, the global APES minimum can be found at $R_{g}$ distance $\left(C_{2 h}\right.$ distortion) from the high-symmetry $D_{3 d}$ geometry while this is $R_{u}$ for the $u$ excited state. $E_{\mathrm{JT}}$, the Jahn-Teller energy, is the energy difference between the $D_{3 d}$ and $C_{2 h}$ configurations. The $\delta_{\mathrm{JT}}$ energy barrier between the three local minima of $\mathrm{C}_{2 h}$ configurations (few meVs) is not shown here for the sake of clarity. In (c), we schematically depict the phonon sideband of the ground and excited state where blue (red) lines represent the excitation (luminescence) of the system into the phonon sideband, whereas the black line defines the zero-phonon line transition (ZPL). The spin-orbit coupling $(\lambda)$ splits ${ }^{2} E_{g}$ and ${ }^{2} E_{u}$ ZPL states apart into $E_{g \frac{1}{2}}, E_{g^{\frac{3}{2}}}, E_{u \frac{1}{2}}, E_{u \frac{3}{2}}$ Kramers doublets, where $\frac{3}{2}$ and $\frac{1}{2}$ refer to an effective total angular momentum of the electronic states. The doublets may further split into individual $e_{g, u \pm}^{\uparrow / \downarrow}$ states under external static magnetic field.

ones. An accurate calculation of the zero-point energies within our DJT treatment together with the spin-orbit coupling brings the computed ZPL energies closer to the experimental ones, with respect to the average ZPL energies. Nevertheless, both methods are accurate within $0.1 \mathrm{eV}$ (see Fig. 2). This gives us confidence that the calculated ZPL energy of $\mathrm{PbV}(-)$ is well predicted. As can be read out from Table I, the nature of ZPL transition involves polaronic states together with spin-orbit effects (see Appendix C). Thus, perturbation effects, e.g., strain or temperature, on ZPL energies should involve the complex analysis of this coupled spin-orbit-phonon system. The calculation of spin-orbit coupling and electron-phonon coupling is described in the following sections.

Because the excited states can be well described by promoting an electron from the $e_{u}$ orbital to the $e_{g}$ orbital according to our CDFT calculations, the optical transition dipole moment for $X \mathrm{~V}(-)$ defects can be well approximated by calculating the optical dipole moment $\mu$ between these Kohn-Sham states. The radiative lifetime of these color centers then can be calculated [55] as

$$
\tau=\frac{n \omega^{3}|\mu|^{2}}{3 \pi \epsilon_{0}}
$$

where $n$ is the refractive index of diamond and $\hbar \omega$ is the excitation energy. The calculated radiative lifetimes are listed and compared to the observed PL lifetime of the
$X \mathrm{~V}(-)$ defects in Table II. A general trend is that the computed radiative lifetime somewhat decreases with increasing atomic number of the impurity atom, but basically they are all in the same order of magnitude. The predicted short radiative lifetime $(\approx 3 \mathrm{~ns})$ of the $\mathrm{PbV}(-)$ center is favorable for quantum emitter applications. We note that the observed PL lifetime of $\mathrm{SiV}(-)$ is significantly shorter than its computed radiative lifetime. We attribute this effect to the strong nonradiative processes which we tentatively assign to the ionization process that competes with the neutral excitation process (see also a recent photoluminescence excitation observation in Ref. [56]). The calculated $(2-\mid-)$ charge transition level of $\mathrm{SiV}(-)$ at $\approx 2.05 \mathrm{eV}$ is very close to the energy of the phonon sidebands of neutral excitation, whereas the energy

TABLE II. The calculated radiative lifetimes $\left(\tau_{\text {rad }}\right)$ versus the observed photoluminescence lifetimes $\left(\tau_{\mathrm{PL}}\right)$ for $X \mathrm{~V}(-)$ color centers at cryogenic temperatures. We use the experimental ZPL energy where available in the calculation of $\tau_{\text {rad }}$. We note that $\tau_{\mathrm{PL}}$ involves both radiative and nonradiative processes.

\begin{tabular}{lcccc}
\hline \hline & $\operatorname{SiV}(-)$ & $\mathrm{GeV}(-)$ & $\mathrm{SnV}(-)$ & $\mathrm{PbV}(-)$ \\
\hline$\tau_{\text {rad }}(\mathrm{ns})$ & 12.13 & 6.62 & 5.49 & 2.88 \\
$\tau_{\mathrm{PL}}(\mathrm{ns})$ & $1.72^{\mathrm{a}}$ & $\sim 6^{\mathrm{b}}$ & $\sim 5^{\mathrm{c}}$ & \\
\hline \hline
\end{tabular}

\footnotetext{
${ }^{\mathrm{a}}$ Reference [57].

${ }^{\mathrm{b}}$ Reference [30].

${ }^{\mathrm{c}}$ Reference [30].
} 
between these two increases with increasing atomic number of the impurity atom that should suppress this type of nonradiative processes. In addition, the optical gap is also significantly larger for $\mathrm{GeV}(-), \operatorname{SnV}(-)$, and $\mathrm{PbV}(-)$ than that of $\mathrm{SiV}(-)$, which also suppresses the direct nonradiative decay induced by phonons in the former color centers.

The phonon sideband in the PL spectrum is determined within the HR theory (see the original theory in Ref. [58] and our implementation in Ref. [59] that is based on Ref. [60]). We calculate the HR spectra between two statically distorted Jahn-Teller structures as depicted in Fig. 3. By this way, we can take into account the contribution of the $e_{g}$ phonons in the PL spectrum that are responsible for the most intense phonon sidebands for $\mathrm{SiV}(-)$ and $\mathrm{GeV}(-)$ defects. These $e_{g}$ phonons are bulklike in nature and do not localize on the defect. The participation of these $e_{g}$ phonons in the phonon sideband is a consequence of the dynamic Jahn-Teller nature of the ground state and excited state. We note that quasilocal vibration modes are also visible as relatively sharp features in the PL spectrum of $\mathrm{SiV}(-)$ and $\mathrm{GeV}(-)$ at about 62 and $43 \mathrm{meV}$, respectively, which are not reproduced by our method. Based on the calculated vibration spectrum of $\mathrm{SiV}(-)$ defect in our previous study [61], we associate this feature with the $e_{u}$ quasilocal vibration modes of the defects that involve the $(x, y)$ motions of the impurity atom. Principally, the usual Franck-Condon approximation on the luminescence of polyatomic systems does not allow the participation of ungerade modes in the PL process; thus, the observations might be explained by invoking the Herzberg-Teller effect that goes beyond the Franck-Condon approximation (see Ref. [61] for details). This issue is beyond the scope of the present study, and we rather focus on the general trends in the PL spectra. As can be seen in Fig. 4 the contribution of phonons to the PL spectrum
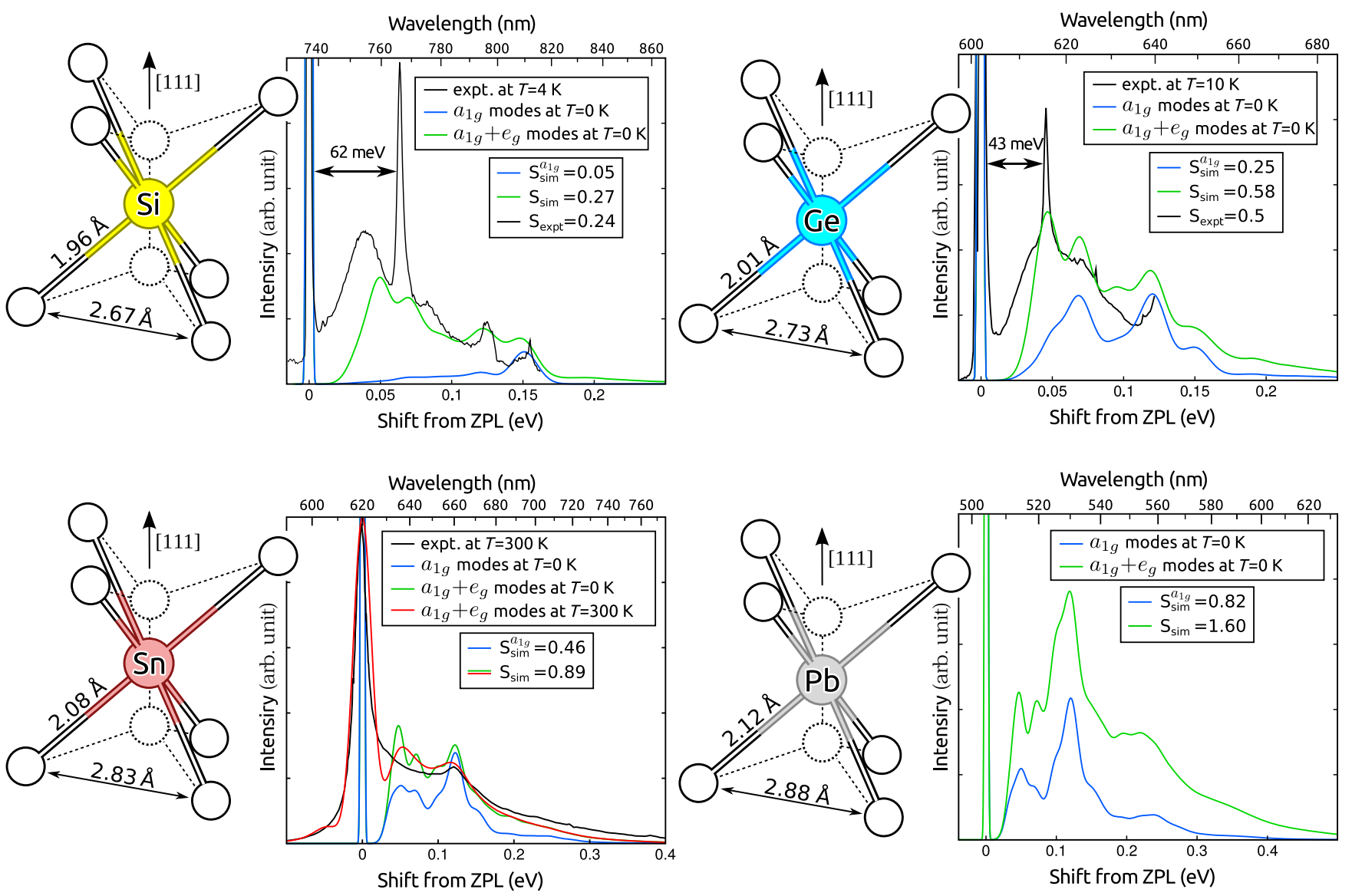

FIG. 4. Experimental and theoretically predicted luminescence line shapes of $X \mathrm{~V}(-)$ complexes. The red, green, and blue curves are theoretical predictions with HR theory, while the black lines are the actual experimental data for $\mathrm{SiV}(-)$ and $\mathrm{GeV}(-)$. The blue curves contain only the effect of totally symmetric $a_{1 g}$ phonons, whereas the green curves add the contribution of $e_{g}$ phonons that appear due to the Jahn-Teller nature of the electronic states. The experimental curve for $\mathrm{SiV}(-)$ and $\mathrm{GeV}(-)$ is taken from Refs. [11,25], respectively. For $\mathrm{SnV}(-)$, we use the 620.3-nm PL spectrum that has been recently recorded in Sn-implanted diamond sample at room temperature (Ref. [34]). We show the room temperature theoretical spectrum for $\mathrm{SnV}$ in the red plot. We set the linewidth of ZPL to match with the experimental data as our present method cannot determine the ZPL broadening. $S$ values are the calculated HR factors taken from our simulations (sim.) or experiments (expt.). 
increases with increasing atomic number of group-IV impurity. In particular, the contribution of $a_{1 g}$ phonons significantly increases because the geometry change between the ground and excited state's geometries increases, as measured by the HR factor $S$. This can be understood by the size of the heavy atoms that cannot readily be accommodated by the divacancy of diamond and they start to substantially distort the diamond lattice. We note that this trend is disadvantageous for creating very efficient single photon sources emitting light dominantly in the zero-phonon emission; nevertheless, the calculated $S=$ 1.60 factor for $\mathrm{PbV}(-)$ is still much smaller than the $S \approx$ 3.5 for $\mathrm{NV}(-)$ center in diamond (see Ref. [45] for detailed analysis).

\section{Effective spin-orbit coupling in $X \mathbf{V}(-)$ defects}

A splitting occurs in both the ${ }^{2} E_{u}$ and ${ }^{2} E_{g}$ levels due to SOC between the $S=1 / 2$ electron spin and the double degenerate orbital forming two Kramers doublets that we call zero-field splitting (ZFS) where zero field refers to zero external magnetic field. In DJT systems, the spin-orbit coupling can reduce the effective SOC by the $p$ Ham reduction factor $[46,62]$. We use here the same $a b$ initio theory to calculate the $p$ factor as we demonstrated for the ${ }^{3} E$ excited state of the negatively charged nitrogen-vacancy center in diamond [45]. This requires us to calculate the full APES in the corresponding electronic state as depicted in Fig. 3. Here we note that $E_{\mathrm{JT}}$ decreases with increasing atomic number of group-IV impurity (see Table III). This results in smaller damping of SOC with increasing atomic number of group-IV impurity. The intrinsic spin-orbit coupling is determined as described in Sec. II. Accurate calculation of spin-orbit coupling requires a scaling method in giant supercells (see Ref. [45] and Appendix A). The results are summarized in Table III.

The general trend is that the $\lambda_{0}$ rapidly grows with increasing atomic number of group-IV impurity. In the ${ }^{2} E_{g}$ ground state, there is a turning point for the $\mathrm{PbV}(-)$ defect where $\lambda_{0}$ is greater than $E_{\mathrm{JT}}$; thus, SOC is not a small perturbation with respect to electron-phonon coupling but the electron-phonon Hamiltonian has to be parallel diagonalized together with the spin-orbit Hamiltonian (see Sec. II and Appendix C). As a consequence, the estimated ZFS between the sublevels of the ${ }^{2} E_{g}$ ground state is around $18.7 \mathrm{meV}$ with the Ham reduction scheme $\left(\lambda_{\mathrm{Ham}}\right)$, and $18.1 \mathrm{meV}(\lambda)$ with the exact diagonalization (see Table III for details). The $p$ Ham reduction parameter also increases with increasing atomic number of group-IV impurity because $E_{\mathrm{JT}}$ decreases, whereas the vacancy-related $\omega_{e}$ vibration modes are relatively insensitive to the type of group-IV impurity atom.

We calculated the $p$ reduction factors for the ${ }^{2} E_{u}$ excited state of the $X \mathrm{~V}(-)$ defects, too (see Table III). In the excited state, the Ham reduction factors are significantly smaller than those in the ground state because the $E_{\mathrm{JT}}$ energies are larger in the excited state than those in the ground state (cf. Table III). We think that the larger electron density in the interstitial region around the impurity atom in the ${ }^{2} E_{u}$ state contributes to form long bonds between the carbon atoms around the impurity atom and thus makes the JahnTeller distorted structure more favorable in that state than in the ${ }^{2} E_{g}$ state. Surprisingly, the Ham factor $p$ scheme provides reasonable results even for the optically excited state of $\mathrm{PbV}(-)$ for which $\lambda_{0}>E_{\mathrm{JT}}$; nevertheless, exact diagonalization of the adjoint DJT and SOC Hamiltonians is needed for accurate results. We show a graphical interpretation for the spin-orbit and electron-phonon

TABLE III. The calculated basic parameters of the APES such as $E_{\mathrm{JT}} \mathrm{Jahn}$-Teller energy, $\delta_{\mathrm{JT}}$ barrier energy, and the $\hbar \omega_{e}$ energy of the effective $e_{g}$ phonon driving the Jahn-Teller effect are shown as well as the calculated $\lambda_{0}$ intrinsic spin-orbit coupling and $p$ Ham reduction factor, and the deduced $\lambda_{\mathrm{Ham}}=p \lambda_{0}$ effective spin-orbit coupling for the ${ }^{2} E_{g}$ optically ground state and ${ }^{2} E_{u}$ excited state of $X \mathrm{~V}(-)$ defects. The calculated zero-field splitting $(\lambda)$ is for comparison to the experimental value $\left(\lambda_{\text {expt }}\right)$. We determine the calculated $\lambda$ values beyond the simple Ham reduction theory where we treat the SOC and DJT Hamiltonians simultaneously (see Fig. 5 for graphical interpretation).

\begin{tabular}{|c|c|c|c|c|c|c|c|c|}
\hline System & $\lambda_{0}(\mathrm{meV}, \mathrm{GHz})$ & $E_{\mathrm{JT}}(\mathrm{meV})$ & $\delta_{\mathrm{JT}}(\mathrm{meV})$ & $\hbar \omega(\mathrm{meV})$ & $p$ & $\lambda_{\text {Ham }}(\mathrm{GHz})$ & $\lambda(\mathrm{GHz})$ & $\lambda_{\text {expt }}(\mathrm{GHz})$ \\
\hline $\operatorname{SiV}\left({ }^{2} E_{g}\right)$ & $0.82,198$ & 42.3 & 3.0 & 85.2 & 0.308 & 61.0 & 61.0 & $50^{\mathrm{a}}$ \\
\hline $\mathrm{GeV}\left({ }^{2} E_{g}\right)$ & $2.20,532$ & 30.1 & 2.0 & 82.2 & 0.390 & 207 & 207 & $181^{\mathrm{b}}$ \\
\hline $\operatorname{SnV}\left({ }^{2} E_{q}\right)$ & $8.28,2001$ & 21.6 & 1.6 & 79.4 & 0.472 & 946 & 945 & $850^{c}$ \\
\hline $\mathrm{PbV}\left({ }^{2} E_{q}\right)$ & $34.6,8360$ & 15.6 & 0.6 & 74.9 & 0.540 & 4514 & 4385 & \\
\hline $\operatorname{SiV}\left({ }^{2} E_{u}\right)$ & $6.96,1680$ & 78.5 & 2.7 & 73.5 & 0.128 & 215 & 215 & $260^{\mathrm{a}}$ \\
\hline $\operatorname{GeV}\left({ }^{2} E_{u}\right)$ & $36.1,8720$ & 85.7 & 5.4 & 73.0 & 0.113 & 987 & 989 & $1120^{\mathrm{b}}$ \\
\hline $\operatorname{SnV}\left({ }^{2} E_{u}\right)$ & $96.8,23200$ & 83.1 & 6.8 & 75.6 & 0.125 & 2897 & 2925 & $3000^{\mathrm{c}}$ \\
\hline $\mathrm{PbV}\left({ }^{2} E_{u}\right)$ & 245,59300 & 91.6 & 12.3 & 78.6 & 0.119 & 7051 & 6920 & \\
\hline
\end{tabular}

\footnotetext{
${ }^{\mathrm{a}}$ Reference [6].

${ }^{\mathrm{b}}$ Reference [25].

${ }^{\mathrm{c}}$ Reference [34].
} 


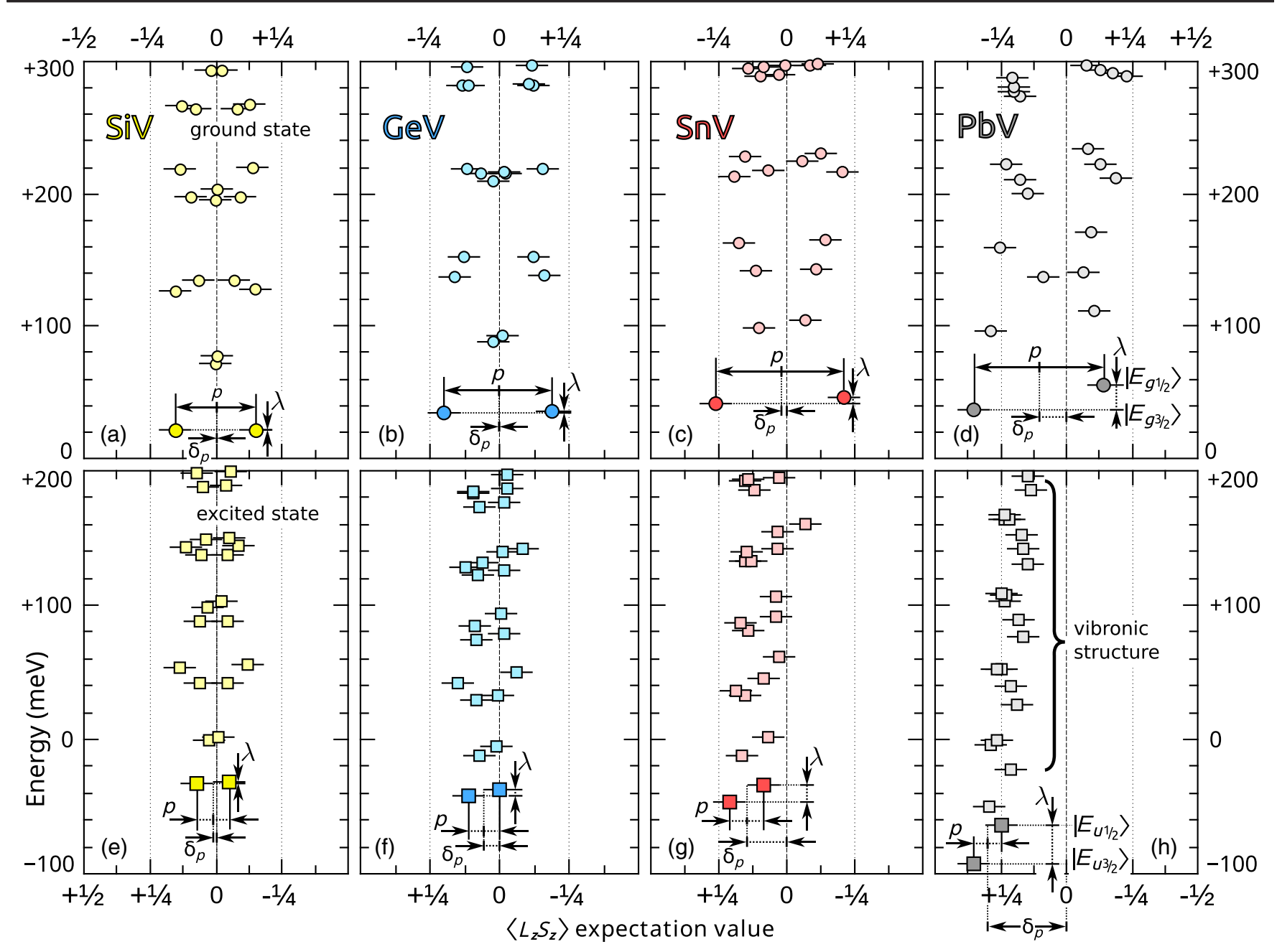

FIG. 5. Calculated eigenvalues of the adjoint DJT [Eq. (4)] and SOC [Eq. (2)] interaction for the $X \mathrm{~V}(-)$ color centers. The two lowest eigenvalues for each figure correspond to the ${ }^{2} E_{g, u}$ vibrionic ground states for $g$ ground (a)-(d) and the optically allowed $u$ excited (e)-(h) states. All the considered eigenvalues are doubly degenerate in spin dimension because of the Kramers degeneracy. Each state consists of pure $\uparrow$ or $\downarrow$ spin state; thus, the fourfold degeneracy of ${ }^{2} E_{g, u}$ is fulfilled. We label the energy difference of the two lowestenergy states by $\lambda$, which is directly observed in the fine structure of the ZPL in the PL spectrum known as zero-field splitting. Along the $x$ axis we depict the eigenvalues with respect to their partially quenched spin-orbit coupling strength $\left\langle L_{z} S_{z}\right\rangle$; thus, one can directly read out the $p$ factors from this figure. $\delta_{p}$ shows deviation of the Ham reduction factors on $E_{g, u \frac{3}{2}}$ and $E_{g, u \frac{1}{2}}$ states. The larger the $\delta_{p}$, the less accurate the treatment of SOC as a perturbation over DJT. We note that a mirror symmetry at $x=0$ shows up for the ground state of SiV center in the entire vibronic spectrum that demonstrates that SOC can be treated as a perturbation over JT effect. The systematic left shift at $x$ axis for the vibronic spectrum of $\mathrm{SnV}$ and $\mathrm{PbV}$ defects implies that $\mathrm{SOC}$ is comparable with the JT coupling.

coupled systems in Fig. 5. We expand the series expansion with the spin degrees of freedom in Eq. (6) then directly diagonalize the Hamiltonian including the SOC and DJT effects simultaneously. As long as $\lambda_{0} \ll E_{\mathrm{JT}}$, SOC is only a small perturbation over the polaronic DJT ground state, and the ${ }^{2} E_{g, u} 4 \times$ degenerate level is split into double degenerate $E_{g, u \frac{3}{2}}$ and $E_{g, u \frac{1}{2}}$ levels. We label this splitting with $\lambda$ in Fig. 5 that is directly observed in the fine structure of the ZPL optical emission. The perturbative approach of SOC is valid mostly for the $\mathrm{SiV}(-)$ system, especially on its optically ground state [Fig. 5(a)]; thus, approximation of $\lambda$ with $\lambda_{\text {Ham }}$ is valid. If SOC energy is higher than $E_{\mathrm{JT}}$, then the Ham reduction scheme still provides surprisingly good results when compared to those from exact diagonalization. Considerable deviations only begin to appear for $\mathrm{PbV}(-)$ (see also Table III). For $\mathrm{SiV}(-)$, the plotted data are symmetric with respect to the $x=0$ axis as the $E_{u \frac{1}{2}}$ is increased by $p \lambda_{0} / 2$ energy and $E_{u_{\frac{3}{2}}}$ is decreased by the same amount. However, for the excited state of $\mathrm{PbV}(-)$, this is not symmetric. The SOC systematically shifts the eigenvalues to the left in $x$ axis. In this case, the $E_{u \frac{1}{2}}$ state contains larger contribution from spin-orbit favored $e_{u-}^{\downarrow}$ and $e_{u+}^{\uparrow}$ states rather than from the unfavorable $e_{u-}^{\uparrow}$ and $e_{u+}^{\downarrow}$ 
states, indicating that the DJT and SOC Hamiltonians should be solved simultaneously.

\section{Spin Hamiltonian for group-IV vacancy qubits and its implications}

By applying an external constant magnetic field, the spin double degenerate levels of the $X \mathrm{~V}(-)$ color centers may split. Previously, a spin Hamiltonian was deduced for a $\mathrm{SiV}(-)$ qubit [6] to describe this feature that we further develop based on the coupled spin-orbit-phonon Hamiltonian for the ground state $(g)$ and excited state (u) as follows:

$$
\begin{aligned}
\hat{\boldsymbol{H}}_{\mathrm{eff}}^{g, u}= & -\underbrace{\left(p^{g, u} \lambda_{0}^{g, u}+K_{\mathrm{JT}}\right)}_{-\lambda^{g, u}} \hat{L}_{z} \hat{S}_{z}+\mu_{B} \underbrace{p^{g, u} g_{L}^{g, u}}_{f^{g, u}} \hat{L}_{z} B_{z} \\
& +\mu_{B} g_{S} \hat{\boldsymbol{S} B}-\underbrace{2 \delta_{p}^{g, u} g_{L}^{g, u}}_{2 \delta_{f}^{g, u}} \hat{S}_{z} B_{z}+\hat{\Upsilon}_{\text {strain }},
\end{aligned}
$$

where $g_{S}=2.0023$ is the $g$ factor of the electron, $\mu_{B}$ is the Bohr magneton of the electron, $\boldsymbol{B}$ is the external homogeneous magnetic field, and $B_{z}$ is its $z$ component with the $z$ axis parallel to the symmetry axis of the defect. We note that the hypefine interaction between the electron spin and nuclear spins in the diamond lattice or with the impurity atom is not considered here. In the braces we merge the different effects into single effective parameters where we follow the nomenclature of Ref. [6] for the two common parameters $\lambda$ and $f$, whereas parameter $\delta_{f}$ appears as a new parameter according to our derivation. Our derivation reveals the microscopic origin of the spin Hamiltonian merged parameters that we discuss below. The operator $\hat{L}_{z}$ acts on the $E_{g, u}$ orbitals as \pm 1 , where $g$ and $u$ refer again to the parity of the wave functions with $g$ the ground state and $u$ the optically allowed excited state.

The derivation of the terms is found in Appendixes $\mathrm{C}$ and D. Here we discuss all the resultant terms in our spin Hamiltonian in detail. The first term in Eq. (9) contains an effective spin-orbit splitting. We first note the negative sign which originates from the three-electron many-body $E_{g}$ and $E_{u}$ states. As a consequence, $E_{g, u \frac{3}{2}}$ is lower in energy than the $E_{g, u \frac{1}{2}}$, in contrast to the previous assignments. Here, we label the states with $m_{j}$ quantum numbers, which is the sum of $m_{l}= \pm 1$ orbital angular momentum and the $m_{s}= \pm \frac{1}{2}$ spin quantum numbers. Since the orbitals are coupled to phonons, the Ham reduction factors $p$ can be different for $\tilde{E}_{g, u \frac{3}{2}}$ and $\tilde{E}_{g, u \frac{1}{2}}$ polaronic wave functions [see Eq. (C8)], and the final $p^{g, u}$ will be the average of the two [see Eq. (C5)]. In addition, the vibronic zero-point energy of these states can also differ, in principle, that will change the energy gap between these two states that we label by $K^{g, u}$ as defined in Eq. (C10). We note that $K^{g, u}$ can be neglected for $\operatorname{SiV}(-)$ and $\mathrm{GeV}(-)$, but it becomes substantial for $\mathrm{SnV}(-)$ and $\mathrm{PbV}(-)$.

In the second term two reduction factors appear. The $p^{g, u}$ is already introduced above and caused by electron-phonon coupling. The $g_{L}^{g, u}$ orbital reduction factor was previously discussed by Stevens [63]; thus, we call it Stevens's orbital reduction factor. This originates from the fact that orbital angular moment $\hat{L}_{z}$ is only an effective operator as the $D_{3 d}$ point group of the $X \mathrm{~V}(-)$ systems does not respect the full $O(3)$ rotational symmetry, as illustrated in Appendix D. We find that the $g_{L}^{g}$ is in particular substantially smaller than one, and significantly reduces the effective $\hat{L}_{z}$ (see Appendix D).

The third term is the usual Zeeman term for electron spins. The fourth term provides a correction to the $g_{S}$ constant; thus, it modifies it to a tensor where the corrected $z z$ component is caused by the polaronic nature of the $\tilde{E}_{g, u \frac{3}{2}}$ and $\tilde{E}_{g, u_{2}^{\frac{1}{2}}}$ states where the $\delta_{p}^{g, u}$ scales with the difference of the corresponding Ham reduction factors [see Eqs. (C9) and (C5) in Appendix C]. This is a new correction which is negligible for $\mathrm{SiV}(-)$ but already appears for the excited state of $\mathrm{GeV}(-)$, and both for the ground and excited state of $\operatorname{SnV}(-)$ and $\mathrm{PbV}(-)$.

We propose that the $\hat{\Upsilon}_{\text {JT }}$ operator in a previous study [6] that was associated with the Jahn-Teller effect originates from the residual strain around the individual $\mathrm{SiV}(-)$ centers; thus, we propose to label it as a $\hat{\Upsilon}_{\text {strain }}$ operator instead. We note that the strain was also considered in that study, which can be very strong (in the order of $100 \mathrm{GHz}$ ) in nanodiamond samples and much smaller in the bulk diamond samples (few $\mathrm{GHz}$ ), which is fairly described in Chaps. 4.1 and 4.3 of Ref. [64]. We find that the strong electron-phonon coupling occurs for the phonons with energy in the order of $10 \mathrm{meV}$ that is clearly manifested in the PL spectrum of $\operatorname{SiV}(-)$. This energy region is significantly larger than the spin-orbit energy, thus the strong electron-phonon coupling, i.e., the DJT effect is manifested via Ham reduction of the spin-orbit coupling. We note that the very low energy acoustic $E_{g}$ phonons might be considered as static strain, which would treat these distortions by static Jahn-Teller effect instead of DJT. In any case, the final form of that Hamiltonian is the strain Hamiltonian; thus, the two effects cannot be distinguished in experiments.

Based on these considerations, we simulate the Zeeman splitting of the corresponding states with a magnetic field aligned to the $\langle 100\rangle$ direction in Fig. 6, where we assume that no strain acts on the $X \mathrm{~V}(-)$ color centers. In these simulations, we used $a b$ initio electron-phonon deduced parameters, but the intrinsic spin-orbit energies $\left(\lambda_{0}\right)$ were scaled in order to reproduce the experimental zero-field splittings $(\lambda)$. We followed this procedure in order to directly compare our results to the experimental spectrum for $\mathrm{SiV}(-), \mathrm{GeV}(-)$, and $\mathrm{SnV}(-)$. The $g_{L}^{g, u}$ parameters 

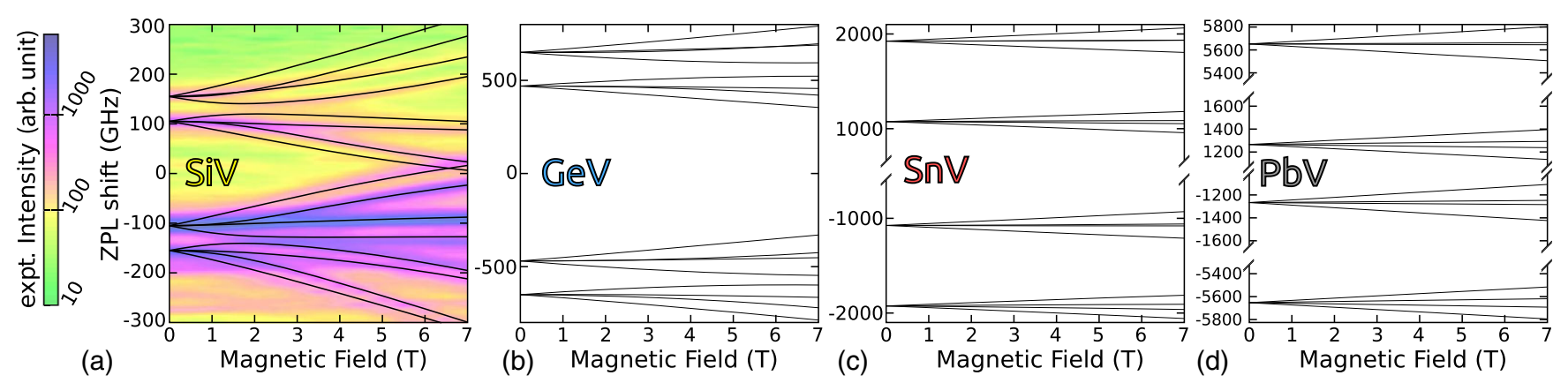

FIG. 6. Magnetic field dependence of the ZPL splitting where the magnetic field is aligned along $\langle 100\rangle$ direction. Zero energy is aligned to no-spin-orbit and no-phonon coupling solution. The $\lambda_{0}$ SOC coupling is fitted to obtain the experimental zero-field splitting for $\mathrm{SiV}$ (a), $\mathrm{GeV}$ (b), and $\mathrm{SnV}$ (c) in order to have a direct comparison to the experimental data whereas full ab initio result is shown for $\mathrm{PbV}$ (d). The actual parameters are listed in Table IV in Appendix C for the effective spin Hamiltonian Eq. (9). We plot all possible transitions between the ${ }^{2} E_{g}$ and ${ }^{2} E_{u}$ states; thus, there are total $4 \times 4$ individual lines for each figure. The plotted emission intensity in (a) is experimental data extracted from Fig. 4.10 on p. 131 in Ref. [64].

were fitted to obtain the experimental spectrum of $\mathrm{SiV}(-)$ and we applied these Stevens's orbital reduction factors for the other $X \mathrm{~V}(-)$ qubits. This is a simple approximation that could lead to slightly underestimated values for the ground state of $\mathrm{GeV}(-), \operatorname{SnV}(-)$, and $\mathrm{PbV}(-)$, where the $d$ orbitals of the impurity atom may contribute to $g_{L}^{g}$. Nevertheless, our simulations should result in relatively accurate spectra. For $\mathrm{SiV}(-)$, our procedure resulted in $g_{L}^{g}=0.328$ and $g_{L}^{u}=0.782$. The strong reduction in the ground state can be understood by the shape of the corresponding orbital that we depict in Fig. 8 in Appendix D. For instance, the $E_{g+}$ state not only transforms as a $m_{l}=+1$ wave function but also as a $m_{l}=-2$ wave function. The linear combinations of the two leads to a significant reduction in the effective interaction with the external magnetic field, i.e., relatively small $g_{L}$. In the $E_{u}$ excited state, the impurity $p$ orbitals contribute to the interaction with the magnetic field unlike in the $E_{g}$ ground state; thus, the reduction parameter is significantly closer to one (no reduction) for the excited state (see Appendix D). The list of all parameters is found in Table IV in Appendix C. For $\mathrm{SiV}(-)$, our spin Hamiltonian can well reproduce the curvatures of the experimental Zeeman spectrum. We apply the same Hamiltonian for the other $X \mathrm{~V}(-)$ qubits. Besides the obvious growing ZFS going from smaller to larger atomic number of impurity atoms, the general trend is that the curves are steeper for larger atomic number of impurity atoms because of the enhanced $g_{z z}$ values, which is caused by the complex spin-orbitphonon coupling [the fourth term in Eq. (9)].

For $\mathrm{PbV}(-)$ the calculated zero-field splitting $\lambda$ in the ${ }^{2} E_{g}$ ground state is about $4.4 \mathrm{THz}$ or $18.2 \mathrm{meV}$, which means that the ${ }^{2} E_{g_{2}^{3}}$ ground state will be thermally filled with $100 \%$ and $\approx 96 \%$ occupation at cryogenic and liquid

TABLE IV. Proposed parameters of the effective Hamiltonian in Eq. (9) for $X \mathrm{~V}(-)$ systems. $\lambda_{0}$ is scaled to reproduce the experimental $\lambda$ for $\mathrm{SiV}(-), \mathrm{GeV}(-)$, and $\mathrm{SnV}(-)$. The $p, \delta_{p}$ reduction parameters are calculated ab initio from the SOC and DJT coupled Hamiltonian (see Fig. 5 for visual interpretation). The orbital $g_{L}$ reduction factors were fit to reproduce the experimental curves for $\mathrm{SiV}(-)$, and the same $g_{L}$ factors were applied to the other $X \mathrm{~V}(-)$ systems. The definition of the parameters shown here can be found in Eqs. (C8)-(C10).

\begin{tabular}{lccccccccccc}
\hline \hline System & $\lambda_{0}(\mathrm{THz})$ & $p_{\frac{3}{2}}$ & $p_{\frac{1}{2}}$ & $p$ & $\delta_{p}$ & $g_{L}$ & $K_{\text {JT }}(\mathrm{GHz})$ & $p \lambda_{0}(\mathrm{GHz})$ & $\lambda(\mathrm{GHz})$ & $f$ & $\delta_{f}$ \\
\hline $\mathrm{SiV}\left({ }^{2} E_{g}\right)$ & 0.163 & 0.311 & 0.305 & 0.308 & 0.003 & 0.328 & 0.06 & 50 & $50^{\mathrm{a}}$ & $0.1^{\mathrm{a}}$ & 0.001 \\
$\mathrm{GeV}\left({ }^{2} E_{g}\right)$ & 0.465 & 0.400 & 0.380 & 0.390 & 0.010 & 0.328 & 0.11 & 181 & $181^{\mathrm{b}}$ & 0.128 & 0.003 \\
$\mathrm{SnV}\left({ }^{2} E_{g}\right)$ & 1.801 & 0.513 & 0.429 & 0.471 & 0.042 & 0.328 & 1.82 & 848 & $850^{\mathrm{c}}$ & 0.154 & 0.014 \\
$\mathrm{PbV}\left({ }^{2} E_{g}\right)$ & 8.361 & 0.705 & 0.284 & 0.494 & 0.211 & 0.328 & 252 & 4133 & $4385^{\mathrm{d}}$ & 0.162 & 0.069 \\
$\mathrm{SiV}\left({ }^{2} E_{u}\right)$ & 2.034 & 0.156 & 0.100 & 0.128 & 0.028 & 0.782 & 0.39 & 260 & $260^{\mathrm{a}}$ & $0.1^{\mathrm{a}}$ & 0.022 \\
$\mathrm{GeV}\left({ }^{2} E_{u}\right)$ & 9.877 & 0.241 & -0.014 & 0.113 & 0.128 & 0.782 & -2.91 & 1123 & $1120^{\mathrm{b}}$ & 0.089 & 0.100 \\
$\mathrm{SnV}\left({ }^{2} E_{u}\right)$ & 23.77 & 0.429 & -0.178 & 0.125 & 0.303 & 0.782 & 18.0 & 2981 & $3000^{\mathrm{c}}$ & 0.098 & 0.238 \\
$\mathrm{PbV}\left({ }^{2} E_{u}\right)$ & 59.30 & 0.709 & -0.500 & 0.105 & 0.604 & 0.782 & 689 & 6231 & $6920^{\mathrm{d}}$ & 0.082 & 0.473 \\
\hline \hline
\end{tabular}

\footnotetext{
${ }^{\mathrm{a}}$ Reference [6].

${ }^{\mathrm{b}}$ Reference [25].

${ }^{\mathrm{c}}$ Reference [34].

${ }^{\mathrm{d}}$ Present $a b$ initio result.
} 
nitrogen temperature, respectively, that can be useful for quantum optics protocols. By applying the theory developed for the estimation of the coherence time of the $\mathrm{SiV}(-)$ (see Ref. [13] and the Supplemental Material in Ref. [34]), we find that the decoherence process caused by the acoustic phonons is completely quenched at cryogenic temperatures for $\mathrm{PbV}(-)$ because of the large $\lambda$ between the two branches of the ${ }^{2} E_{g}$ ground state, and the coherence time of the electron spin should be limited only by the nuclear spins or electron spins in the diamond crystal. That is much more practical for quantum communication applications compared to the millikelvin cooling needed for similar coherence time in a $\mathrm{SiV}(-)$ qubit [14]. We note that the energy gap in $\operatorname{SnV}(-)$ should result in microsecond to millisecond coherence time for the electron spin going from 4 to $1 \mathrm{~K}$ measurement temperature [34].

\section{SUMMARY AND CONCLUSION}

We perform a systematic study on the magneto-optical properties of group-IV vacancy color centers in diamond by means of $a b$ initio density functional theory calculations. We identify the photostability of these centers that can act as solid-state qubits. We develop a novel spin Hamiltonian for these qubits in which the electron angular momentum and spin as well as the phonons are strongly coupled, and identify such terms that have not been considered so far but are important in understanding their magneto-optical properties. We solve $a b$ initio this complex problem for the model of these color centers consisting of up to 1000-atom supercells, and are able to reproduce previous experimental data. Furthermore, we identify $\operatorname{SnV}(-)$ and $\mathrm{PbV}(-)$ qubits with long spin coherence time at cryogenic temperatures where the spin state of $\mathrm{PbV}(-)$ can also be thermally initialized at these temperatures. Our $a b$ initio tool kit and spin Hamiltonian analysis serve as a template for similar studies in 3D materials such as silicon carbide or 2D materials such as hexagonal boron nitride or transitional metal dichalgonides (TMD) or dioxides (TMO), which are fast emerging materials hosting qubits or single photon sources. In particular, the TMD and TMO materials exhibit strong spin-orbit couplings induced by the transition metal ions in the crystal in which strong mixing of spin-orbit and electron-phonon coupling are expected in the defects acting as qubits, and they should be treated on equal footing.

\section{ACKNOWLEDGMENTS}

Support from ÚNKP-17-3-III New National Excellence Program of the Ministry of Human Capacities of Hungary, the National Research Development and Innovation Office of Hungary within the Quantum Technology National Excellence Program (Project Contract No. 2017-1.2.1NKP-2017-00001), and the EU Commission (DIADEMS Project Contract No. 611143) is acknowledged.

\section{APPENDIX A: CONVERGENCE OF SPIN-ORBIT ENERGIES AS A FUNCTION OF SUPERCELL SIZE}

Here we provide the calculated spin-orbit energies for the ground $(g)$ and excited $(u)$ state as a function of the size of the supercell within $\Gamma$-point sampling of the Brillouin zone by the HSE06 DFT functional. We determine the $\lambda_{0}$ intrinsic SOC parameters by fitting the $\lambda_{0}(L)=$ $\lambda_{0}+A \exp (-L \cdot B)$ function on the data points as obtained from 216-, 512-, and 1000-atom diamond supercells (see Fig. 7). Here $L$ is the length of the corresponding cubic diamond supercell and $\lambda_{0}, A$, and $B$ are the fitting parameters. We also calculate the effective SOC for the

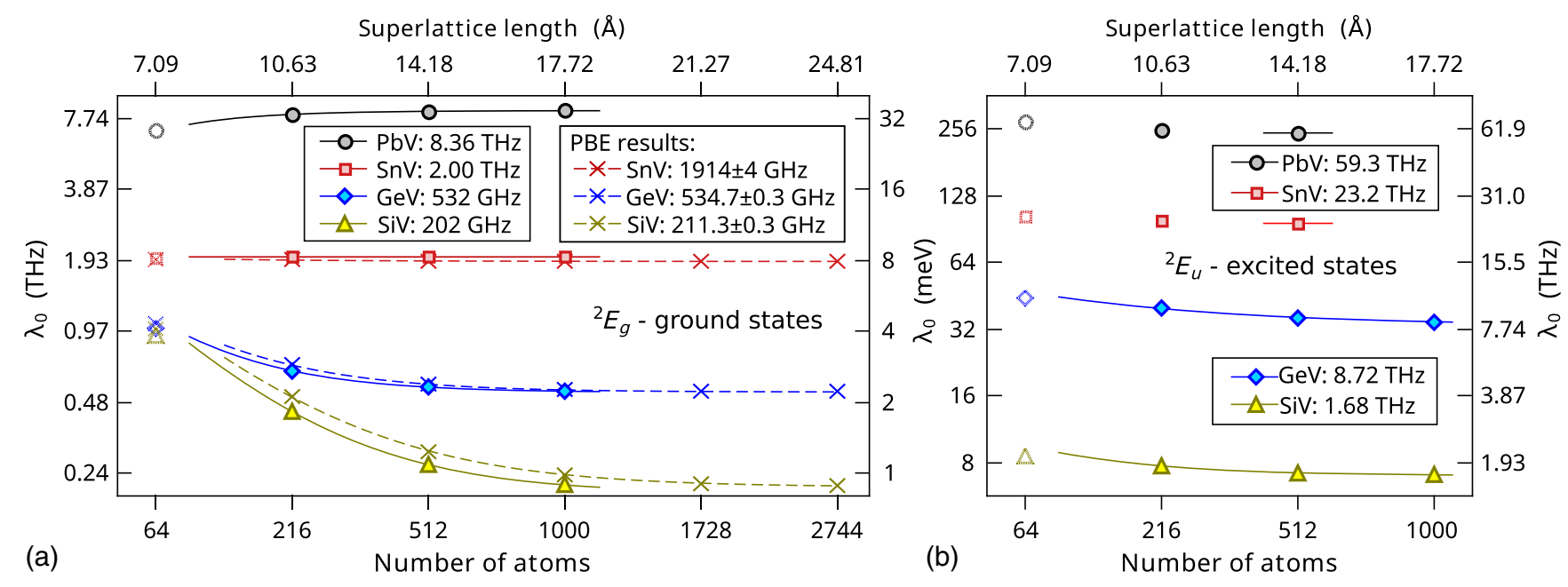

FIG. 7. Calculated spin-orbit energies with HSE06 DFT functional as a function of the size of the supercell within $\Gamma$-point approximation for (a) ${ }^{2} E_{g}$ ground and (b) ${ }^{2} E_{u}$ excited state. The converged values are given in the label boxes. We show the computationally less expensive PBE results for giant supercells for ${ }^{2} E_{g}$ ground state, too. The 5 data points for PBE yield a standard error for the fitting. The small standard error in these calculations supports the exponential decay for HS06 data points. 
optically active ${ }^{2} E_{u}$ excited states where the calculation procedure goes as described for the $E_{g}$ state in Sec. II. We note that the 512-atom supercell is proven to be convergent for ${ }^{2} E_{u}$ spin-orbit energies in $\mathrm{SnV}(-)$ and $\mathrm{PbV}(-)$ defects; thus, we use those energies as isolated converged values. We prove the exponential decay of SOC parameters by the computationally less demanding Perdew-Burke-Ernzerhof [44] calculations that allows us to use giant supercells in the scaling study (see Fig. 7).

\section{APPENDIX B: DERIVATION OF THE HAM REDUCTION FACTOR}

Here we briefly derive the Ham reduction factor for the cases where the spin-orbit coupling is significantly smaller than the electron-phonon interaction, and it can be treated as a first order perturbation. We start with the definition of the electronic orbitals in either the real or imaginary forms:

$$
\left|e_{g \pm}\right\rangle=\frac{1}{\sqrt{2}}\left(\left|e_{x}\right\rangle \pm i\left|e_{y}\right\rangle\right)=\frac{1}{\sqrt{2}}\left(\begin{array}{c}
1 \\
\pm i
\end{array}\right) \leftarrow\left|e_{x}\right\rangle .
$$

The vibronic wave function $\tilde{\Psi}_{ \pm}$caused by electron-phonon interaction can be expanded as written in Eq. (6), which mixes the electronic orbitals and $|n, m\rangle e_{g}$ phonons. The spin-orbit coupling then should be calculated for the $\tilde{\Psi}_{ \pm}$ wave function:

$$
\lambda_{\mathrm{Ham}}=2\left|\left\langle\tilde{\Psi}_{ \pm}\left|\hat{H}_{\mathrm{SOC}}\right| \tilde{\Psi}_{ \pm}\right\rangle\right|
$$

By expanding $\tilde{\Psi}_{ \pm}$in Eq. (B2), we arrive at

$$
\sum_{n, m, k, l}\left[c _ { n m } \left\langlee _ { g \pm } \left|\left\langlen, m\left|+d_{n m}\left\langle e_{g \mp}|\langle n, m|] \hat{H}_{\mathrm{SOC}}\left[c_{k l}\left|e_{g \pm}\right\rangle|k, l\rangle+d_{k l}\left|e_{g \mp}\right\rangle|k, l\rangle\right],\right.\right.\right.\right.\right.\right.
$$

which leads to

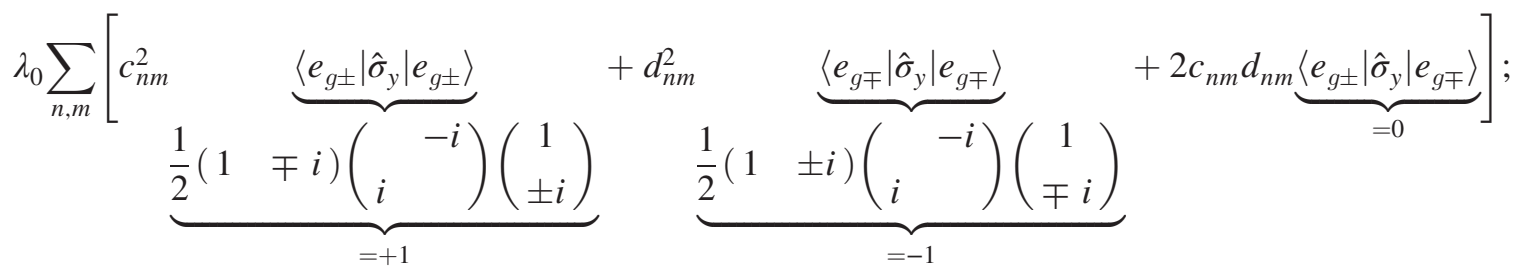

that is,

$$
\lambda_{0} \sum_{n, m}\left[c_{n m}^{2}-d_{n m}^{2}\right]=\lambda_{0} p
$$

The final formula in Eq. (B5) defines the Ham reduction factor $p$.

\section{APPENDIX C: DERIVATION OF THE EFFECTIVE HAMILTONIAN}

In Eq. (6) the lower-energy $\left(\frac{3}{2}\right)$ and higher-energy $\left(\frac{1}{2}\right)$ states split by spin-orbit interaction share the same $p$ reduction factors in their corresponding ground and excited state. However, the $\left\langle L_{z} S_{z}\right\rangle$ weights are not symmetric, as shown in Fig. 5, due to the electron-phonon coupling; thus, we introduce individual Hamiltonians for each doublet $\left(\Gamma=E_{g^{\frac{1}{2}}}, E_{g_{\frac{3}{2}}^{3}}, E_{u \frac{1}{2}}, E_{u \frac{3}{2}}\right)$ in the following,

$\hat{H}_{\text {eff }}^{\Gamma}=|\Gamma\rangle\langle\Gamma|\left[p_{\Gamma}^{g, u} \hat{L}_{z}\left(-\lambda_{0}^{g, u} \hat{S}_{z}+g_{L}^{g, u} B_{z}\right)+g_{S} \hat{\boldsymbol{S}} \boldsymbol{B}\right]$,

where $|\Gamma\rangle\langle\Gamma|$ projector ensures that we stay in the $\Gamma$ doublet in Eq. (C1). The projectors can be expressed by spin-orbit operators as

$$
\begin{aligned}
& \left|E_{g, u \frac{3}{2}}\right\rangle\left\langle E_{g, u \frac{3}{2}}\right|=\frac{1}{2}+\hat{L}_{z} \hat{S}_{z}, \\
& \left|E_{g, u \frac{1}{2}}\right\rangle\left\langle E_{g, u \frac{1}{2}}\right|=\frac{1}{2}-\hat{L}_{z} \hat{S}_{z} .
\end{aligned}
$$

We note that as $|\Gamma\rangle\langle\Gamma|$ commutes with other parts of $\hat{H}^{\Gamma}$, one needs to include the projector only once in Eq. (C1). The actual $p_{\Gamma}$ values can be directly read out from Fig. 5, which we define as

$$
\begin{aligned}
& p_{\frac{3}{2}}^{g, u}=+2\left\langle\tilde{E}_{g, u \frac{3}{2}}\left|\hat{L}_{z} \hat{S}_{z}\right| \tilde{E}_{g, u \frac{3}{2}}\right\rangle, \\
& p_{\frac{1}{2}}^{g, u}=-2\left\langle\tilde{E}_{g, u \frac{1}{2}}\left|\hat{L}_{z} \hat{S}_{z}\right| \tilde{E}_{g, u \frac{1}{2}}\right\rangle .
\end{aligned}
$$

These parameters are the expectation value of the (dimensionless) SOC acted on the electron-phonon coupled wave functions. $\tilde{E}_{g, u \frac{1}{2}}$ and $\tilde{E}_{g, u \frac{3}{2}}$ are the wave functions from series expansion of Eq. (7) of the adjoint SOC and DJT interaction. In the next step, we unify the $\frac{3}{2}$ and $\frac{1}{2}$ substates inside the ground and excited state manifold, 
$\hat{H}_{\mathrm{eff}}^{g, u}=\sum_{\Gamma=E_{g, u \frac{1}{2}}, E_{g, u \frac{3}{2}}} \hat{H}_{\mathrm{eff}}^{\Gamma}=\left(\frac{1}{2}-\hat{L}_{z} \hat{S}_{z}\right) p_{\frac{1}{2}}^{g, u} \hat{L}_{z}\left(-\lambda_{0 g, u} \hat{S}_{z}+g_{L}^{g, u} B_{z}\right)+\left(\frac{1}{2}+\hat{L}_{z} \hat{S}_{z}\right) p_{\frac{3}{2}}^{g, u} \hat{L}_{z}\left(-\lambda_{0}^{g, u} \hat{S}_{z}+g_{L}^{g, u} B_{z}\right)+g_{S} \hat{\boldsymbol{S}} \boldsymbol{B}$,

where we can take advantage of the properties of $\hat{L}_{z}$ and $\hat{S}_{z}$ to arrive at

$$
\hat{H}_{\mathrm{eff}}^{g, u}=\underbrace{\frac{1}{2}\left(p_{\frac{3}{2}}^{g, u}+p_{\frac{1}{2}}^{g, u}\right)}_{=p_{g, u}} \hat{L}_{z}\left(-\lambda_{0 g, u} \hat{S}_{z}+g_{L}^{g, u} B_{z}\right)+\underbrace{\left(p_{\frac{3}{2}}^{g, u}-p_{\frac{1}{2}}^{g, u}\right) \lambda_{0}^{g, u}}_{\text {offset }} \underbrace{\hat{L}_{z}^{2} \hat{S}_{z}^{2}}_{\frac{1}{4}}-\underbrace{\left(p_{\frac{3}{2}}^{g, u}-p_{\frac{1}{2}}^{g, u}\right) g_{L}^{g, u}}_{=2 \delta_{g, u}^{f}} \hat{S}_{z} B_{z} \underbrace{L_{z}^{2}}_{1}+g_{S} \hat{\boldsymbol{S} \boldsymbol{B} .}
$$

Here we substitute $\hat{L}_{z}^{2}=1$ and $\hat{S}_{z}^{2}=\frac{1}{4}$ for $X \mathrm{~V}(-)$ systems. The offset term in Eq. (C5) does not depend on the magnetic field or cause splitting between the branches of the ground and excited state; therefore, it is not observable in the experiments. That is rather a correction term for the firstprinciples calculations after turning on the spin-orbit and electron-phonon couplings. We use this correction in the calculation of the ZPL energies. Next, we consider only the observable terms in the spin Hamiltonian derivation for $X \mathrm{~V}(-)$ color centers, which goes as

$\hat{H}_{\mathrm{eff}}^{g, u}=-\lambda^{g, u} L_{z} S_{z}+f^{g, u} L_{z} B_{z}+g_{S} S \boldsymbol{B}-2 \delta_{f}^{g, u} S_{z} B_{z}$,

where we introduce the following parameters:

$$
\lambda^{g, u}=p^{g, u} \lambda_{0}^{g, u}+K_{\mathrm{JT}}^{g, u}, \quad f^{g, u}=p^{g, u} g_{L}^{g, u}, \quad \delta_{f}^{g, u}=\delta_{p}^{g, u} g_{L}^{g, u} .
$$

The actual parameters can be calculated from the DJT and SOC entangled wave functions, i.e., the $E_{g, u \frac{3}{2}}$ and $E_{g, u \frac{1}{2}}$ doublets, as

$$
\begin{aligned}
p^{g, u}= & \frac{p_{\frac{3}{2}}^{g, u}+p_{\frac{1}{2}}^{g, u}}{2}=\left\langle\tilde{E}_{g, u_{\frac{3}{2}}}\left|\hat{L}_{z} \hat{S}_{z}\right| \tilde{E}_{g, u \frac{3}{2}}\right\rangle \\
& +\left\langle\tilde{E}_{g, u_{2}}\left|\hat{L}_{z} \hat{S}_{z}\right| \tilde{E}_{g, u_{2}}\right\rangle
\end{aligned}
$$

and

$$
\begin{aligned}
\delta_{p}^{g, u}= & \frac{p_{\frac{3}{2}}^{g, u}-p_{\frac{1}{2}}^{g, e}}{2}=\left\langle\tilde{E}_{g, u \frac{2}{2}}\left|\hat{L}_{z} \hat{S}_{z}\right| \tilde{E}_{g, u \frac{3}{2}}\right\rangle \\
& -\left\langle\tilde{E}_{g, u \frac{1}{2}}\left|\hat{L}_{z} \hat{S}_{z}\right| \tilde{E}_{g, u \frac{1}{2}}\right\rangle .
\end{aligned}
$$

The term $K_{\mathrm{JT}}^{g, u}$ requires further explanation. This comes from the fact that the zero-point energy of the vibronic ${ }^{2} E_{g, u \frac{3}{2}}$ and ${ }^{2} E_{g, u \frac{1}{2}}$ wave functions is not the same; thus, this can cause an extra splitting labeled by $K_{\mathrm{JT}}^{g, u}$ that adds to $p^{g, u} \lambda^{g, u} \cdot K_{\mathrm{JT}}^{g, u}$ can be calculated as

$$
\begin{aligned}
K_{\mathrm{JT}}^{g, u}= & \left\langle\tilde{E}_{g, u \frac{1}{2}}\left|\hat{H}_{\mathrm{DJT}}\right| \tilde{E}_{g, u \frac{1}{2}}\right\rangle+ \\
& -\left\langle\tilde{E}_{g, u \frac{3}{2}}\left|\hat{H}_{\mathrm{DJT}}\right| \tilde{E}_{g, u \frac{3}{2}}\right\rangle .
\end{aligned}
$$

This term gives negligible correction to the ZFS of $\mathrm{GeV}(-)$ and $\mathrm{SiV}(-)$ but becomes significant for $\mathrm{PbV}(-)$, as shown in Table IV.

\section{APPENDIX D: ORIGIN OF STEVENS'S ORBITAL REDUCTION FACTOR}

The orbital angular momentum of an atomic orbital may be reduced in the potential created by surrounding ions that reduce the spherical symmetry [63]. We show that similar quenching can take place for vacancy-type defects in diamond. In the particular case of $X \mathrm{~V}(-)$ color centers, the carbon dangling bonds in the vacancies form double degenerate $E_{g \pm}$ and $E_{u \pm}$ states. The $E_{g, u \pm}$ states were considered as $m_{l}= \pm 1$ states [6,64], which led to the assumption of $g_{L}^{g, u}= \pm 1$. However, one should note that the $E_{g, u}$ state will also transform as $m_{l}=\mp 2$ under $D_{3 d}$ symmetry. We illustrate this by plotting the $e_{g, u \pm}$ orbitals in comparison to the $m_{l}= \pm 1$ and $m_{l}=\mp 2$ wave functions in Fig. 8. It can be observed that the $e_{g, u \pm}$ orbitals can be rather described as $\alpha\left|m_{l}= \pm 1\right\rangle+\sqrt{1-\alpha^{2}}\left|m_{l}=\mp 2\right\rangle$, where $\alpha$ is a coefficient of the $m_{l}= \pm 1$ contribution. This results in a $\left|g_{L}\right|$ that is smaller than 1. Since the $p$

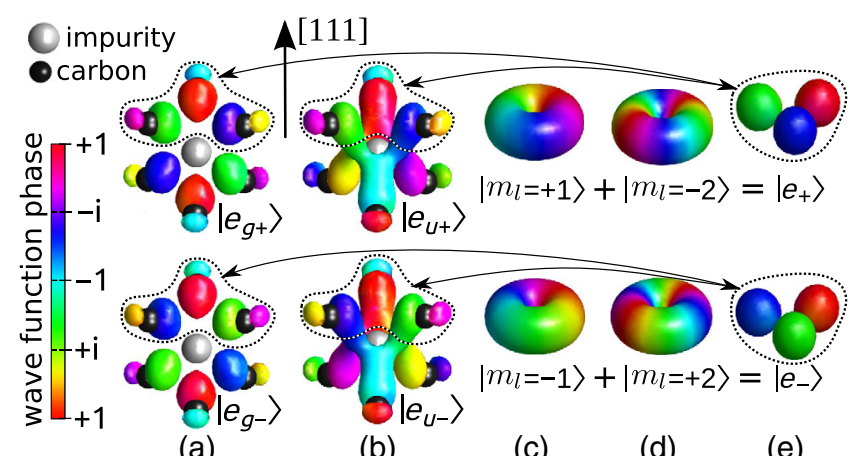

(a)

(b)

(c)

(d)

(e)

FIG. 8. Isosurfaces represent the phase of (a) $e_{g \pm}$ orbitals, (b) $e_{u \pm}$ orbitals of $\mathrm{SiV}(-)$ qubit from side view ( $z$ axis parallel to [111] direction), (c) $m_{l}= \pm 1$ wave functions, and (d) $m_{l}=\mp 2$ wave functions. Linear combinations of $(c)+(d)$ (admixtures of atomic $n=2, l=1 m_{l}= \pm 1$ and $n=3 l=2 m_{l}=\mp 2$ orbitals) can result in (e) $e_{+}$or $e_{-}$orbitals very similar to that obtained in (a) and (b). The sign of the wave function does not change for $(g)$ orbitals, whereas it changes for $(u)$ orbitals upon inversion. 
orbitals of the group-IV atom can contribute to $m_{l}= \pm 1$ in the $E_{u}$ excited state, in contrast to the case of $E_{g}$ ground state, $g_{L}^{g}$ is smaller than $g_{L}^{u}$ in $X \mathrm{~V}(-)$ qubits. We note that the full $a b$ initio calculation of the expectation value of $\hat{L}_{z}$ is not straightforward. The implementation of spin-orbit coupling in VASP is based on the assumption that the spin-orbit interaction is predominant close to the core of the atoms and it is negligible in the interstitial regions, which is considered to be a well-justified approximation. In that case, the spin-orbit integrals can be distributed to the spherical volumes (PAW spheres) around the ions with using the atomic wave function projectors and potential. However, as Fig. 8 demonstrates, the interstitial regions can significantly contribute to the expectation value of $\hat{L}_{z}$, which gives the interaction between the electron wave function and the external magnetic field. Therefore, we rather did not estimate this quantity from the values in the PAW sphere of the ions.

[1] A. M. Zaitsev, V. S. Vavilov, and A. A. Gippius, Cathodoluminescence of Diamond Associated with Silicon Impurity, Sov. Phys. Lab. Inst. Rep. 10, 15 (1981).

[2] J. P. Goss, R. Jones, S. J. Breuer, P. R. Briddon, and S. Öberg, The Twelve-Line $1.682 \mathrm{eV}$ Luminescence Center in Diamond and the Vacancy-Silicon Complex, Phys. Rev. Lett. 77, 3041 (1996).

[3] C. D. Clark, H. Kanda, I. Kiflawi, and G. Sittas, Silicon Defects in Diamond, Phys. Rev. B 51, 16681 (1995).

[4] A. Gali and J. R. Maze, Ab Initio Study of the Split SiliconVacancy Defect in Diamond: Electronic Structure and Related Properties, Phys. Rev. B 88, 235205 (2013).

[5] E. Neu, D. Steinmetz, J. Riedrich-Möller, S. Gsell, M. Fischer, M. Schreck, and C. Becher, Single Photon Emission from Silicon-Vacancy Colour Centres in Chemical Vapour Deposition Nano-Diamonds on Iridium, New J. Phys. 13, 025012 (2011).

[6] C. Hepp, T. Müller, V. Waselowski, J. N. Becker, B. Pingault, H. Sternschulte, D. Steinmüller-Nethl, A. Gali, J. R. Maze, M. Atatüre, and C. Becher, Electronic Structure of the Silicon Vacancy Color Center in Diamond, Phys. Rev. Lett. 112, 036405 (2014).

[7] L. J. Rogers, K. D. Jahnke, M. H. Metsch, A. Sipahigil, J. M. Binder, T. Teraji, H. Sumiya, J. Isoya, M. D. Lukin, P. Hemmer, and F. Jelezko, All-Optical Initialization, Readout, and Coherent Preparation of Single Silicon-Vacancy Spins in Diamond, Phys. Rev. Lett. 113, 263602 (2014).

[8] A. Sipahigil, K. D. Jahnke, L. J. Rogers, T. Teraji, J. Isoya, A. S. Zibrov, F. Jelezko, and M. D. Lukin, Indistinguishable Photons from Separated Silicon-Vacancy Centers in Diamond, Phys. Rev. Lett. 113, 113602 (2014).

[9] B. Pingault, D.-D. Jarausch, C. Hepp, L. Klintberg, J. N. Becker, M. Markham, C. Becher, and M. Atatüre, Coherent Control of the Silicon-Vacancy Spin in Siamond, Nat. Commun. 8, 15579 (2017).

[10] B. Pingault, J. N. Becker, C. H. H. Schulte, C. Arend, C. Hepp, T. Godde, A. I. Tartakovskii, M. Markham,
C. Becher, and M. Atatüre, All-Optical Formation of Coherent Dark States of Silicon-Vacancy Spins in Siamond, Phys. Rev. Lett. 113, 263601 (2014).

[11] A. Dietrich, K. D. Jahnke, J. M. Binder, T. Teraji, J. Isoya, L. J. Rogers, and F. Jelezko, Isotopically Varying Spectral Features of Silicon-Vacancy in Diamond, New J. Phys. 16, 113019 (2014).

[12] J. N. Becker, J. Görlitz, C. Arend, M. Markham, and C. Becher, Ultrafast All-Optical Coherent Control of Single Silicon Vacancy Colour Centres in Diamond, Nat. Commun. 7, 13512 (2016).

[13] K. D. Jahnke, A. Sipahigil, J. M. Binder, M. W. Doherty, M. Metsch, L. J. Rogers, N. B. Manson, M. D. Lukin, and F. Jelezko, Electron-Phonon Processes of the Silicon-Vacancy Centre in Diamond, New J. Phys. 17, 043011 (2015).

[14] D. D. Sukachev, A. Sipahigil, C. T. Nguyen, M. K. Bhaskar, R. E. Evans, F. Jelezko, and M. D. Lukin, Silicon-Vacancy Spin Qubit in Diamond: A Quantum Memory Exceeding 10 ms with Single-Shot State Readout, Phys. Rev. Lett. 119, 223602 (2017).

[15] J. N. Becker, B. Pingault, D. Groß, M. Gündogan, N. Kukharchyk, M. Markham, A. Edmonds, M. Atatüre, P. Bushev, and C. Becher, All-Optical Control of the SiliconVacancy Spin in Diamond at Millikelvin Temperatures, Phys. Rev. Lett. 120, 053603 (2018).

[16] C. T. Nguyen, R. E. Evans, A. Sipahigil, M. K. Bhaskar, D. D. Sukachev, V. N. Agafonov, V. A. Davydov, L. F. Kulikova, F. Jelezko, and M. D. Lukin, All-Optical Nanoscale Thermometry with Silicon-Vacancy Centers in Diamond, arXiv:1708.05419.

[17] U. F. S. D'Haenens-Johansson, A. M. Edmonds, B. L. Green, M. E. Newton, G. Davies, P. M. Martineau, R. U. A. Khan, and D. J. Twitchen, Optical Properties of the Neutral Silicon Split-Vacancy Center in Diamond, Phys. Rev. B 84, 245208 (2011).

[18] B. C. Rose, D. Huang, Z.-H. Zhang, A. M. Tyryshkin, S. Sangtawesin, S. Srinivasan, L. Loudin, M. L. Markham, A. M. Edmonds, D. J. Twitchen, S. A. Lyon, and N. P. de Leon, Observation of an Environmentally Insensitive Solid State Spin Defect in Diamond, arXiv:1706.01555.

[19] K. Iakoubovskii and A. Stesmans, Characterization of Hydrogen and Silicon-Related Defects in CVD Diamond by Electron Spin Resonance, Phys. Rev. B 66, 195207 (2002).

[20] A. M. Edmonds, M. E. Newton, P. M. Martineau, D. J. Twitchen, and S. D. Williams, Electron Paramagnetic Resonance Studies of Silicon-Related Defects in Diamond, Phys. Rev. B 77, 245205 (2008).

[21] T. Iwasaki, F. Ishibashi, Y. Miyamoto, Y. Doi, S. Kobayashi, T. Miyazaki, K. Tahara, K. D. Jahnke, L. J. Rogers, B. Naydenov, F. Jelezko, S. Yamasaki, S. Nagamachi, T. Inubushi, N. Mizuochi, and M. Hatano, GermaniumVacancy Single Color Centers in Diamond, Sci. Rep. 5, 12882 (2015).

[22] V. G. Ralchenko, V.S. Sedov, A. A. Khomich, V.S. Krivobok, S. N. Nikolaev, S. S. Savin, I. I. Vlasov, and V. I. Konov, Observation of the Ge-Vacancy Color Center in Microcrystalline Diamond Films, Bull. Lebedev Phys. Inst. 42, 165 (2015). 
[23] S. Häußler, G. Thiering, A. Dietrich, N. Waasem, T. Teraji, J. Isoya, T. Iwasaki, M. Hatano, F. Jelezko, A. Gali, and A. Kubanek, Photoluminescence Excitation Spectroscopy of $\mathrm{SiV}$ - and GeV-Color Center in Diamond, New J. Phys. 19, 063036 (2017).

[24] Y. N. Palyanov, I. N. Kupriyanov, Y. M. Borzdov, and N. V. Surovtsev, Germanium: A New Catalyst for Diamond Synthesis and a New Optically Active Impurity in Diamond, Sci. Rep. 5, 14789 (2015).

[25] E. A. Ekimov, S. G. Lyapin, K. N. Boldyrev, M. V. Kondrin, R. Khmelnitskiy, V. A. Gavva, T. V. Kotereva, and M. N. Popova, Germanium-Vacancy Color Center in Isotopically Enriched Diamonds Synthesized at High Pressures, JETP Lett. 102, 701 (2015).

[26] Y. N. Palyanov, I. N. Kupriyanov, A. F. Khokhryakov, and Y. M. Borzdov, High-Pressure Crystallization and Properties of Diamond from Magnesium-Based Catalysts, CrystEngComm 19, 4459 (2017).

[27] P. Siyushev, M. H. Metsch, A. Ijaz, J. M. Binder, M. K. Bhaskar, D. D. Sukachev, A. Sipahigil, R. E. Evans, C. T. Nguyen, M. D. Lukin, P. R. Hemmer, Y. N. Palyanov, I. N. Kupriyanov, Y. M. Borzdov, L. J. Rogers, and F. Jelezko, Optical and Microwave Control of Germanium-Vacancy Center Spins in Diamond, Phys. Rev. B 96, 081201 (2017).

[28] E. A. Ekimov, V. S. Krivobok, S. G. Lyapin, P. S. Sherin, V. A. Gavva, and M. V. Kondrin, Anharmonicity Effects in Impurity-Vacancy Centers in Diamond Revealed by Isotopic Shifts and Optical Measurements, Phys. Rev. B 95, 094113 (2017).

[29] J. P. Goss, P. R. Briddon, M. J. Rayson, S. J. Sque, and R. Jones, Vacancy-Impurity Complexes and Limitations for Implantation Doping of Diamond, Phys. Rev. B 72, 035214 (2005).

[30] M. K. Bhaskar, D. D. Sukachev, A. Sipahigil, R. E. Evans, M. J. Burek, C. T. Nguyen, L. J. Rogers, P. Siyushev, M. H. Metsch, H. Park, F. Jelezko, M. Lončar, and M. D. Lukin, Quantum Nonlinear Optics with a Germanium-Vacancy Color Center in a Nanoscale Diamond Waveguide, Phys. Rev. Lett. 118, 223603 (2017).

[31] V. Nadolinny, A. Komarovskikh, Y. Palyanov, I. Kupriyanov, Y. Borzdov, M. Rakhmanova, O. Yuryeva, and S. Veber, EPR Study of Si- and Ge-Related Defects in HPHT Diamonds Synthesized from Mg-Based Solvent-Catalysts, Phys. Status Solidi A 213, 2623 (2016).

[32] A. Komarovskikh, A. Dmitriev, V. Nadolinny, and Y. Palyanov, A DFT Calculation of EPR Parameters of a Germanium-Vacancy Defect in Diamond, Diam. Relat. Mater. 76, 86 (2017).

[33] S. D. Tchernij, T. Herzig, J. Forneris, J. Küpper, S. Pezzagna, P. Traina, E. Moreva, I. P. Degiovanni, G. Brida, N. Skukan, M. Genovese, M. Jakšić, J. Meijer, and P. Olivero, Single-Photon-Emitting Optical Centers in Diamond Fabricated upon Sn Implantation, ACS Photonics 4, 2580 (2017).

[34] T. Iwasaki, Y. Miyamoto, T. Taniguchi, P. Siyushev, M. H. Metsch, F. Jelezko, and M. Hatano, Tin-Vacancy Quantum Emitters in Diamond, Phys. Rev. Lett. 119, 253601 (2017).

[35] G. Kresse and J. Furthmüller, Efficient Iterative Schemes for Ab Initio Total-Energy Calculations Using a Plane-Wave Basis Set, Phys. Rev. B 54, 11169 (1996).
[36] P. E. Blöchl, Projector Augmented-Wave Method, Phys. Rev. B 50, 17953 (1994).

[37] O. Bengone, M. Alouani, P. Blöchl, and J. Hugel, Implementation of the Projector Augmented-Wave LDA + U Method: Application to the Electronic Structure of NiO, Phys. Rev. B 62, 16392 (2000).

[38] A. Gali, Identification of Individual ${ }^{13} \mathrm{C}$ Isotopes of Nitrogen-Vacancy Center in Diamond by Combining the Polarization Studies of Nuclear Spins and First-Principles Calculations, Phys. Rev. B 80, 241204 (2009).

[39] J. Heyd, G. E. Scuseria, and M. Ernzerhof, Hybrid Functionals Based on a Screened Coulomb Potential, J. Chem. Phys. 118, 8207 (2003).

[40] A. V. Krukau, O. A. Vydrov, A. F. Izmaylov, and G. E. Scuseria, Influence of the Exchange Screening Parameter on the Performance of Screened Hybrid Functionals, J. Chem. Phys. 125, 224106 (2006).

[41] P. Deák, B. Aradi, T. Frauenheim, E. Janzén, and A. Gali, Accurate Defect Levels Obtained from the HSE06 RangeSeparated Hybrid Functional, Phys. Rev. B 81, 153203 (2010).

[42] S. Lany and A. Zunger, Assessment of Correction Methods for the Band-Gap Problem and for Finite-Size Effects in Supercell Defect Calculations: Case Studies for $\mathrm{ZnO}$ and GaAs, Phys. Rev. B 78, 235104 (2008).

[43] G. Makov and M. C. Payne, Periodic Boundary Conditions in Ab Initio Calculations, Phys. Rev. B 51, 4014 (1995).

[44] J. P. Perdew, K. Burke, and M. Ernzerhof, Generalized Gradient Approximation Made Simple, Phys. Rev. Lett. 77, 3865 (1996).

[45] G. Thiering and A. Gali, Ab Initio Calculation of Spin-Orbit Coupling for an NV Center in Diamond Exhibiting Dynamic Jahn-Teller Effect, Phys. Rev. B 96, 081115 (2017).

[46] F. S. Ham, Dynamical Jahn-Teller Effect in Paramagnetic Resonance Spectra: Orbital Reduction Factors and Partial Quenching of Spin-Orbit Interaction, Phys. Rev. 138, A1727 (1965).

[47] I. Bersuker, The Jahn-Teller Effect (Cambridge University Press, Cambridge, England, 2006).

[48] I. Bersuker and V. Polinger, Vibronic Interactions in Molecules and Crystals, Vol. 49 (Springer Science \& Business Media, Berlin, 2012).

[49] G. Auböck, J. Nagl, C. Callegari, and W. E. Ernst, Observation of Relativistic $E \otimes e$ Vibronic Coupling in Rb3 and K3 Quartet States on Helium Droplets, J. Chem. Phys. 129, 114501 (2008).

[50] P. Mondal, D. Opalka, L. V. Poluyanov, and W. Domcke, Ab Initio Study of Dynamical E $\times$ e Jahn-Teller and SpinOrbit Coupling Effects in the Transition-Metal Trifluorides $\mathrm{TiF}_{3}, \mathrm{CrF}_{3}$, and $\mathrm{NiF}_{3}$, J. Chem. Phys. 136, 084308 (2012).

[51] Y. N. Palyanov, I. N. Kupriyanov, Y. M. Borzdov, A. F. Khokhryakov, and N. V. Surovtsev, High-Pressure Synthesis and Characterization of Ge-Doped Single Crystal Diamond, Cryst. Growth Des. 16, 3510 (2016).

[52] A. T. Collins, L. Allers, C. J. H. Wort, and G. A. Scarsbrook, The Annealing of Radiation Damage in De Beers Colourless CVD Diamond, Diam. Relat. Mater. 3, 932 (1994).

[53] E. Neu, D. Steinmetz, J. Riedrich-Möller, S. Gsell, M. Fischer, M. Schreck, and C. Becher, Single Photon Emission from Silicon-Vacancy Colour Centres in Chemical Vapour Deposition Nano-Diamonds on Iridium, New J. Phys. 13, 025012 (2011). 
[54] L. J. Rogers, K. D. Jahnke, M. W. Doherty, A. Dietrich, L. P. McGuinness, C. Müller, T. Teraji, H. Sumiya, J. Isoya, N. B. Manson, and F. Jelezko, Electronic Structure of the Negatively Charged Silicon-Vacancy Center in Diamond, Phys. Rev. B 89, 235101 (2014).

[55] V. Weisskopf and E. Wigner, Berechnung der Natürlichen Linienbreite auf Grund der Diracschen Lichttheorie, Z. Phys. 63, 54 (1930).

[56] S. Häßler, G. Thiering, A. Dietrich, N. Waasem, T. Teraji, J. Isoya, T. Iwasaki, M. Hatano, F. Jelezko, A. Gali, and A. Kubanek, Photoluminescence Excitation Spectroscopy of $\mathrm{SiV}^{-}$and $\mathrm{GeV}^{-}$Color Center in Diamond, New J. Phys. 19, 063036 (2017).

[57] L. J. Rogers, K. D. Jahnke, T. Teraji, L. Marseglia, C. Müller, B. Naydenov, H. Schauffert, C. Kranz, J. Isoya, L. P. McGuinness, and F. Jelezko, Multiple Intrinsically Identical Single-Photon Emitters in the Solid State, Nat. Commun. 5, 4739 (2014).

[58] K. Huang and A. Rhys, Theory of Light Absorption and Non-Radiative Transitions in F-Centres, Proc. R. Soc. A 204, 406 (1950).
[59] A. Gali, T. Demján, M. Vörös, G. Thiering, E. Cannuccia, and A. Marini, Electron-Vibration Coupling Induced Renormalization in the Photoemission Spectrum of Diamondoids, Nat. Commun. 7, 11327 (2016).

[60] A. Alkauskas, B. B. Buckley, D. D. Awschalom, and C. G. Van de Walle, First-Principles Theory of the Luminescence Lineshape for the Triplet Transition in Diamond NV Centres, New J. Phys. 16, 073026 (2014).

[61] E. Londero, G. Thiering, A. Gali, and A. Alkauskas, Vibrational Modes of Negatively Charged Silicon-Vacancy Venters in Diamond from Ab Initio Calculations, arXiv:1605.02955.

[62] F. S. Ham, Effect of Linear Jahn-Teller Coupling on Paramagnetic Resonance in $a{ }^{2} E$ State, Phys. Rev. 166, 307 (1968).

[63] K. W. H. Stevens, On the Magnetic Properties of Covalent XY 6 Complexes, Proc. R. Soc. A 219, 542 (1953).

[64] C. J. Hepp, Electronic Structure of the Silicon Vacancy Color Center in Diamond, Ph.D. thesis, Saarland University, 2014. 